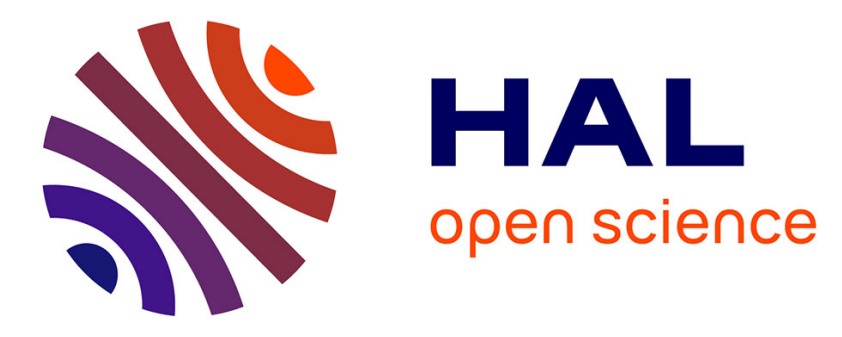

\title{
Tracking the degradation pathway of three model aqueous pollutants in a heterogeneous Fenton process
}

Mélanie Cohen, Nassira Ferroudj, Audrey Combes, Valérie Pichon, Sébastien

Abramson

\section{To cite this version:}

Mélanie Cohen, Nassira Ferroudj, Audrey Combes, Valérie Pichon, Sébastien Abramson. Tracking the degradation pathway of three model aqueous pollutants in a heterogeneous Fenton process. Journal of Environmental Chemical Engineering, 2019, 7, pp.102987 -. 10.1016/j.jece.2019.102987 . hal03486287

\section{HAL Id: hal-03486287 \\ https://hal.science/hal-03486287}

Submitted on 20 Dec 2021

HAL is a multi-disciplinary open access archive for the deposit and dissemination of scientific research documents, whether they are published or not. The documents may come from teaching and research institutions in France or abroad, or from public or private research centers.
L'archive ouverte pluridisciplinaire HAL, est destinée au dépôt et à la diffusion de documents scientifiques de niveau recherche, publiés ou non, émanant des établissements d'enseignement et de recherche français ou étrangers, des laboratoires publics ou privés.

\section{(ㅇ)(1) $\$$}

Distributed under a Creative Commons Attribution - NonCommerciall 4.0 International 


\title{
Tracking the degradation pathway of three model aqueous pollutants in a
}

\section{heterogeneous Fenton process using high resolution mass spectrometry combined}

\author{
with other analytical methods
}

Mélanie Cohen ${ }^{1}$, Nassira Ferroudj², Audrey Combes ${ }^{1}, V^{2}$ alérie Pichon ${ }^{1,3}$, Sébastien Abramson*4

${ }^{1}$ : Dept of Analytical, Bioanalytical Sciences and Miniaturization (LSABM), CNRS, ESPCI Paris, PSL Research University, 10 rue Vauquelin, 75231 Paris Cedex 05, France

2: Laboratoire de Génie Chimique et Environnement de Skikda, Université du 20 Août 1955 de Skikda, BP 26 Route d'El Hadaiek, Skikda, Algeria

${ }^{3}$ : Sorbonne Université, 4 Place Jussieu, 75005 Paris, France

${ }^{4}$ : Laboratoire de PHysico-chimie des Electrolytes et Nanosystèmes InterfaciauX (PHENIX UMR 7195, UPMCCNRS), Faculté des Sciences et Ingénierie, Sorbonne Université, Campus Pierre et Marie Curie, 4 place Jussieu, 75252 Paris Cedex 05, France

* : corresponding author - sebastien.abramson@ sorbonne-universite.fr

Keywords: Fenton reaction, Heterogeneous catalysis, Advanced oxidation mechanism, Quadrupoletime-of-flight mass spectrometry, Analytical chemistry

\footnotetext{
Abstract

The degradation pathway of three model aqueous pollutants, methyl orange, methylene blue, and paranitrophenol, in a Fenton process based on the use of maghemite/silica microspheres as magnetically separable heterogeneous catalyst was investigated. The utilization of different analytical methods, 
including UV-visible spectroscopy, non-purgeable organic carbon (NPOC) measurements, quadrupoletime-of-flight high resolution mass spectrometry (Qtof-HRMS), liquid chromatography coupled with mass spectrometry (LC-MS), and ion chromatography (IC) allowed the identification at different times of many transformation products for each pollutants-pollutant. Theses-These analyses confirmed first the existence of intermediates which have been already evidenced in previous studies on the advanced oxidation of these pollutants. However, new products were also identified, particularly thanks to the highly accurate Qtof-HRMS analyses. The evolution with time of the main intermediate compounds was monitored by LC-MS. It was then possible to propose an overall transformation pathway for each pollutant. The same classes of reactions, especially ring-hydroxylations and N-demethylations, were observed for the three pollutants, although specific features were also pointed out. While some common features with other advanced oxidations processes were found, the highlighted specificities could be related to the selection of the catalyst and to the experimental conditions used for the degradation tests. 


\section{Introduction}

Advanced Oxidation Processes (AOPs) are a set of chemical methods for the treatment of effluents, based on the oxidation by some in-situ generated hydroxyl $\left(\mathrm{HO}^{\circ}\right)$ radicals. This highly reactive species is characterized by one of the largest reduction potentials $\left(\mathrm{E}^{0}=2.8 \mathrm{~V}\right)$, and can easily react with numerous organic molecules. Therefore AOPs have been widely used for the removal of organic pollutants in air, water and soils [1-3]. AOPs were found to be particularly promising for wastewater treatment, with two main applications: (i) the treatment of highly charged industrial or agricultural effluents containing toxic compounds recalcitrant to biodegradation such as dyes, pesticides, or persistent organic pollutants (POPs) [4], In such applications, AOPs is generally applied before the biodegradation step, and instead of the complete mineralization of the pollutants, a partial oxidation leading to more biodegradable and less toxic organic compounds can be obtained.(ii) the removal of small amount of micropollutants such as pesticides, drug residues or detergents, at the end of the treatment sequence. (tertiary or quaternary treatment). Indeed, the usual physicochemical or biological methods are inoperative to remove these emerging classes of contaminants, which frequently behave like endocrine disfuptors, or have other toxic effects-[5]. In that case, a-complete mineralization of the pollutants can be aimed, leading to environmentally benign mineral species such as $\mathrm{CO}_{2}, \mathrm{H}_{2} \mathrm{O}$, and inorganic salts.

AOPs are classified as a function of the production mode of the $\mathrm{HO}^{\bullet}$ radicals, which can be generated by various procedures, including radiolysis, photochemistry, electrochemistry, sonochemistry, or purely chemical methods [6]. In the case of the Fenton type processes, the formation of $\mathrm{HO}^{\circ}$ results from the addition of hydrogen peroxide $\left(\mathrm{H}_{2} \mathrm{O}_{2}\right)$, in combination with a homøgenous homogeneous or heterogeneous catalyst. Although other transition metals can also be used, The the Fenton catalysts are often based on iron, since this element gives the best eompromises compromise in terms of activity, cost and safety [7]. Fenton type processes have been shown to be one of the most efficient methods for the removal of organic pollutants, with respect to other AOPs [8]. Additional advantages offered by these processes are the possibility to work in the absence of light, and the relatively low costs of the reagent and catalyst [8-10]. Over the last three decades, increasing work has been carried out on the replacement of the traditional homogeneous Fenton catalysts based on soluble iron (II) or (III), by heterogeneous 
Fenton catalysts containing iron oxide particles or supported iron ions, owing to the following advantages [11-14]: (i) The heterogeneous catalysts can be recovered and reused, which reduces the formation of secondary wastes resulting from the catalyst (iron sludge's sludges). (ii) There is a possibility to work at a-neutral $\mathrm{pH}$, increasing the range of efficiency of this process, since homogenous homogeneous Fenton catalysts are only active at acidic $\mathrm{pH}$.

In this study, we have employed maghemite/silica $\left(\gamma-\mathrm{F}_{z} \Theta_{3} / \mathrm{SiO} \mathrm{O}_{z} \quad \gamma-\mathrm{Fe}_{2} \mathrm{O}_{3} / \mathrm{SiO}_{2}\right)$ nanocomposite microspheres as heterogeneous Fenton catalyst, in the continuity of our previous works [15]. The use of this catalyst was motivated by the easiness of its separation from the effluent, due to its high magnetic susceptibility. Indeed this material can be easily recovered by magnetically assisted settlement, through the application of a magnetic field gradient obtained with a magnet or electromagnet. This can considerably decrease the cost of its recovery and recycling. We determined the activity of this catalyst in a large range of conditions, on three model pollutants, methyl orange (MO), methylene blue (MB) and paranitrophenol (PNP). These three compounds were chosen because they are good models of wellknown classes of organic aqueous pollutants. Thus, MO belongs to the vast class of azo dyes, some of which are earcinogens-carcinogenic and mutagenic, while PNP is an example of the recalcitrant and toxic phenolic compounds, present in numerous industrial effluents. $\mathrm{MB}$, which is used in various biomedical applications, can be toxic and ecotoxic at high doses [16-18]. Furthermore, these pollutants differ in their charge. At acid or neutral $\mathrm{pH}, \mathrm{MO}$ has an anionic charge, MB is cationic, and PNP is neutral. We have evidenced that the $\gamma \mathrm{F}_{2} \mathrm{O}_{3} / \mathrm{SiO}_{2} \gamma-\mathrm{Fe}_{2} \mathrm{O}_{3} / \mathrm{SiO}_{2}$ catalyst is highly active in the Fenton oxidation of these three compounds, with complete decolorizations of the pellutants-pollutant solutions in few hours. However, relatively low mineralization yields were obtained, indicating that unidentified organic byproducts were formed during the oxidation of the pollutants. In addition, we have evidenced strong differences in reactivity for these three compounds, the rates of decolorization and mineralization strongly varying, although similar conditions were used. This led us to question how these compounds are decomposed. The aim of this study is therefore to highlight the similarities and differences in the degradation pathway pathways of these compounds, to explain their difference in reactivity. 
Simultaneously, we want to compare the degradation mechanisms with those which have been described for other AOPs.

There have been a huge number of publications about the removal of these three model pollutants by AOPs, especially by the Fenton-type processes. In the majority of these papers, the efficiency of the process is simply evaluated through the measurement of the decolorization and/or the mineralization of the pollutant solutions, without focusing on the intermediates-intermediate compounds resulting from the degradation. However it has been shown that the transformation byproducts can be sometimes more toxic or more stable than the parent compound, and frequently remain in small amount at the end of the reaction, having a detrimental impact on the practicability of the process $[19,20]$. The degradation pathways of MO, MB and PNP have been studied only for a relatively small number of AOPs including photocatalysis [21-30], photolysis [31-32], radiolysis [33-34], plasma and direct anodic oxidation [3538], homogeneous and heterogeneous Fenton [39-41], photo-Fenton [42-44], electro-Fenton [45-46], and sono-Fenton [47] processes. Additional work is therefore required for other systems, to specify what it is common or different, with regard to the existing degradation pathways. In addition, some of the previously published studies have been carried out without a thorough work on the identification of the intermediate compounds: the authors merely used the $\mathrm{m} / \mathrm{z}$ value of the molecular ions obtained by LC MS or GC MS analysis, and by comparison with previous studies, proposed an identification of the intermediates.

Here, a complete work on the identification of the intermediate compounds resulting from the heterogeneous Fenton degradation of the three model pollutants (MO, MB, and PNP) is carried out, though-through the complementary use of several techniques. In particular, the samples have been analysed analyzed by quadrupole-time-of-flight high resolution mass spectrometry (Qtof-HRMS). This technology is able to determine with a high accuracy the mass of the parent ions-high accuracy the masses of the parent ions (MS1 mode) and the corresponding fragment ions (MS2 mode), allowing an unambiguous identification of the main constituents of the mixture. HRMS has been rarely employed in the studies on the AOPs mechanisms [20,48-51]. The use of this technique, in combination with more usual methods such as liquid chromatography coupled with mass spectrometry (LC-MS), ion 
chromatography (IC), UV-visible spectroscopy, and non-purgeable organic carbon (NPOC) measurements enabled us to propose a degradation pathway for the three pollutants, and to highlight the presence of intermediate compounds, which had not yet been observed, or whose existence had to be confirmed. In the next sections, the results of the analyses will be only detailed for MO, since a similar approach was taken for MB and PNP. For these two other compounds, the presentation will be limited to the main degradation intermediates and to the global degradation pathways (the complete analyses can be found in the SI part).

\section{Experimental part}

\section{2-1. Catalyst synthesis and characterization}

The synthesis of the $\gamma-\mathrm{Fe}_{2} \mathrm{O}_{3} / \mathrm{SiO}_{2}$ catalyst has been already described in our previous paper [15]. Briefly, magnetite $\left(\mathrm{Fe}_{3} \mathrm{O}_{4}\right)$ NP were first prepared by the Massart's method, adding ammonia to an aqueous mixture of $\mathrm{FeCl}_{3}$ and $\mathrm{FeCl}_{2}$ [52]. Then, the $\mathrm{Fe}_{3} \mathrm{O}_{4} \mathrm{NP}$ were oxidized to $\gamma$ - $\mathrm{Fe}_{2} \mathrm{O}_{3}$ by successively adding $\mathrm{HNO}_{3}$ and $\mathrm{Fe}\left(\mathrm{NO}_{3}\right)_{3}$. The $\gamma-\mathrm{Fe}_{2} \mathrm{O}_{3} \mathrm{NP}$ were finally obtained as an aqueous acidic dispersion with a high colloidal stability, also called ferrofluid. The $\gamma-\mathrm{Fe}_{2} \mathrm{O}_{3} \mathrm{NP}$ were supported on the surface of porous silica microspheres via a protocol inspired from the method originally described by Andersson et al. [53]. A magnetic sol containing the $\gamma-\mathrm{Fe}_{2} \mathrm{O}_{3} \mathrm{NP}$ and a silica precursor (Tetraethoxysilane, TEOS) in acidic medium was added dropwise to an organic phase composed of a vegetable oil and a commercial emulsifier (Arlacel P135 from Uniquema), under stirring. The water-in-oil emulsion thus formed was transferred into a Büchner flask, and the ethanol formed by the hydrolysis of TEOS was evaporated from the dispersed phase under reduced pressure, which led to a rapid condensation of TEOS in silica in each water droplet. The emulsion was then broken by addition of a large amount of acetone. The catalytic beads were washed several times with acetone and water, and dried at $70{ }^{\circ} \mathrm{C}$. Finally, they were calcined in an oven under air at $400^{\circ} \mathrm{C}$ for $18 \mathrm{~h}$ to remove any organic traces.

Transmission electron microscopy (TEM) micrographs of the catalyst were taken using a JEM JEOL $100 \mathrm{CX}$ microscope operating at $100 \mathrm{kV}$. In this aim, the sample was embedded in a resin (AGAR 100), 
which was then polymerised at $60^{\circ} \mathrm{C}$ during two days, and cut in $70 \mathrm{~nm}$ thin sections using a LEICA ULTRACUT UCT microtome apparatus. The catalyst was also observed by scanning electron microscopy (SEM) on a SEM-FEG Hitachi SU-70 apparatus. The images were taken in secondary electron mode with an accelerating voltage of $10 \mathrm{kV}$. Prior to analysis, the beads were coated with a thin shell of gold by sputter deposition. The amount of $\gamma-\mathrm{Fe}_{2} \mathrm{O}_{3} \mathrm{NP}$ in the silica beads was determined by spectrophotometry. In this aim, a given weight of material was first introduced in an $\mathrm{HCl}$ aqueous solution $\left(4 \mathrm{~mol} \mathrm{~L}^{-1}\right)$. After $48 \mathrm{~h}$ of stirring, the $\gamma-\mathrm{Fe}_{2} \mathrm{O}_{3} \mathrm{NP}$ were totally dissolved, and the iron concentration in the supernatant was analyzed by atomic absorption spectrophotometry (Perkin Elmer AA100 apparatus) and the weight fraction of $\gamma-\mathrm{Fe}_{2} \mathrm{O}_{3}$ in the beads, $\mathrm{w}_{\mathrm{Fe} 2 \mathrm{O} 3}$ was determined. X-ray diffractograms of the pure $\gamma-\mathrm{Fe}_{2} \mathrm{O}_{3} \mathrm{NP}$, and $\gamma-\mathrm{Fe}_{2} \mathrm{O}_{3} / \mathrm{SiO}_{2}$ sample were recorded using a Phillips PW 1130 diffractometer. Data were collected from $2 \theta=10^{\circ}$ to $80^{\circ}$ in $0.1^{\circ}$ steps. The experimental conditions for the other methods utilized to characterize the catalyst (SQUID magnetometry, UV-VisNIR spectroscopy, $\mathrm{N}_{2}$ and $\mathrm{CO}_{2}$ adsorption volumetry) can be found in reference [15].

\section{2-2. Catalytic tests}

The activity of the $\gamma-\mathrm{Fe}_{2} \mathrm{O}_{3} / \mathrm{SiO}_{2}$ catalyst was tested for the degradation of methyl orange (MO), methylene blue (MB), or paranitrophenol (PNP). All the experiments were carried out in the dark at a mild temperature $\left(\mathrm{T}=40^{\circ} \mathrm{C}\right)$, in a closed vessel to avoid evaporation. First, $0.3 \mathrm{~g}$ of catalyst, (equivalent to $[\mathrm{Fe}]_{\mathrm{T}}=3.15 \times 10^{-2} \mathrm{~mol} \mathrm{~L}^{-1}$ in the final reaction mixture) was-were added to an aqueous solution of the pollutant, which ( $\mathrm{pH}$ was previously adjusted at 3 by addition of $\mathrm{HNO}_{3}$ ). The volume of this solution was chosen in a manner that the initial concentration of the pollutant in the final mixture was $\mathrm{C}_{\mathrm{i}}=2.5 \times 10^{-}$ ${ }^{4} \mathrm{~mol} \mathrm{~L}^{-1}$ for a total volume of $10 \mathrm{~mL}$ (except for the MB pollutant, where $\mathrm{C}_{\mathrm{i}}$ was fixed at $8 \times 10^{-4} \mathrm{~mol} \mathrm{~L}^{-1}$ to take into account the much larger adsorption of this compound on the silica surface). The suspension containing the catalysts and the pollutant was magnetically stirred for $2 \mathrm{~h}$ to reach the adsorption equilibrium. Then, $0.68 \mathrm{~mL}$ of a $30 \% \mathrm{w} / \mathrm{w} \mathrm{H}_{2} \mathrm{O}_{2}$ aqueous solution was-were added to the suspension (corresponding to a concentration of $\mathrm{H}_{2} \mathrm{O}_{2}$ of $0.68 \mathrm{~mol} \mathrm{~L}^{-1}$ in the final mixture). The initial time $\mathrm{t}=0$ was fixed at this moment. 
The decolorization kinetic-kinetics in the supernatant was monitored by the following procedure. Solution samples were taken at desired time intervals, and were put over a magnet to separate the supernatant from the catalysts by magnetic settlement. The supernatant was then recovered and diluted to an adequate concentration before to be analysed-analyzed with an UV-Visible spectrophotometer (UVIKON XL apparatus). The remaining color in the supernatant was determined by measuring the absorbance $\mathrm{A}$ at $\lambda=502,665$, and $400 \mathrm{~nm}$ respectively for $\mathrm{MO}, \mathrm{MB}$, and PNP. The decolorization rate is given by the ratio $100 \times \mathrm{A} / \mathrm{A}_{0}$, where $\mathrm{A}_{0}$ is the color of the supernatant measured at $\mathrm{t}=0$. The mineralization kinetic-kinetics in the supernatant was determined by a similar procedure, analyzing the non-purgeable organic carbon at desired time intervals (NPOC, in ppm) using a Shimadzu TOC ASI5000A apparatus. Several reproducibility tests were carried out, and it was determined that the percentage of uncertainty on NPOC measurements is $+/-12.5 \%$. The mineralization rate is given by the ratio $100 \times N P O C / N P O C$, , where $\mathrm{NPOC}_{i}$ is the initial NPOC concentration (before the adsorption equilibrium). $\mathrm{NPOC}_{\mathrm{i}}$ was chosen as reference, to take into account the decrease of NPOC at $\mathrm{t}=0$, due to the initial adsorption of the pollutant on the catalyst. Taking into account that for a ratio the relative uncertainties are added, the relative uncertainties on $\mathrm{NPOC} / \mathrm{NPOC}_{\mathrm{i}}$ were estimated to be $+/-25 \%$. The iron leaching was determined by measuring the iron concentrations in the supernatant using atomic absorption spectrophotometry. The percentage of leached iron leached -was calculated by $100 \times[\mathrm{Fe}]_{\mathrm{S}} /[\mathrm{Fe}]_{\mathrm{T}}$ where $[\mathrm{Fe}]_{\mathrm{s}}$ is the iron concentration in the supernatant at $\mathrm{t}$, and $[\mathrm{Fe}]_{\mathrm{T}}$ the equivalent iron concentration in the catalytic test $\left([\mathrm{Fe}]_{\mathrm{T}}=3.15 \times 10^{-2} \mathrm{~mol} \mathrm{~L}^{-1}\right)$.

\section{2-3. LC-MS analysis}

$500 \mu \mathrm{L}$ of the supernatant issued of the degradation solution were taken after 2 minutes, 30 minutes, 60 minutes, 2 hours, 4 hours (for MO and MB only), 6 hours (for MB only), 8 hours (for PNP only), and 24 hours of reaction, and immediately $500 \mu \mathrm{L}$ of acetonitrile were added. The resulting solution was directly injected in LC/MS-MS. The analyses were performed using a reversed phase liquid chromatograph (UltiMate 3000®, Thermo Scientific, Illkirch, France) coupled with a Triple Stage

Quadrupole Mass Spectrometer (TSQ Quantum Access MAX, Thermo Scientific, Illkirch, France) equipped with a heated electrospray ionization source (HESI2). Chromatographic separations were 
conducted on a Sunfire C18 column $(150 \times 2.1 \mathrm{~mm}, 5 \mu \mathrm{m})$ at $28^{\circ} \mathrm{C}$. The flow rate and the injection volume were respectively set at $0.2 \mathrm{~mL} \mathrm{~min}^{-1}$ and $5 \mu \mathrm{L}$. Mobile phases were water containing $0.1 \%(\mathrm{v} / \mathrm{v})$ formic acid (A) and acetonitrile containing $0.1 \%(\mathrm{v} / \mathrm{v})$ formic acid (B) for $\mathrm{MO}$, or methanol containing $0.1 \%(\mathrm{v} / \mathrm{v})$ formic acid for $\mathrm{MB}$ and PNP $(\mathrm{C})$. For MO, the separation was performed in isocratic mode with a mixture of $\mathrm{A}$ and $\mathrm{B}, 50 / 50$, v/v as mobile phase. For $\mathrm{MB}$, we used a linear gradient from 10 to $60 \%$ of $\mathrm{C}$ in $20 \mathrm{~min}$, then the percentage of $\mathrm{C}$ increase up to $90 \%$ in $5 \mathrm{~min}$, hold for 3 minutes, ramp over in 2 min to $10 \% \mathrm{C}$ and hold for $10 \mathrm{~min}$ to equilibrate the system. For PNP, we used a linear gradient from 10 to $70 \%$ of $\mathrm{C}$ in $30 \mathrm{~min}$, ramp over $2 \mathrm{~min}$ to $10 \% \mathrm{C}$ and hold for $10 \mathrm{~min}$ to equilibrate the system.

MS was operated in positive ion mode for MB and negative ion mode for PNP and MO using an electrospray voltage of $3000 \mathrm{~V}$ and $2500 \mathrm{~V}$ respectively. Capillary and vaporizer temperatures were respectively set at $270^{\circ} \mathrm{C}$ and $300^{\circ} \mathrm{C}$ in negative mode, and at $320^{\circ} \mathrm{C}$ and $50^{\circ} \mathrm{C}$ in positive mode. In both modes, nitrogen was used as desolvatation gas. The instrument was operated in full scan mode (Q3) in positive and negative ion mode $(\mathrm{m} / \mathrm{z}=50-350)$ in order to identify intermediates intermediate compounds issued effrom the degradation. SIM mode was also performed in order to follow the kinetics of the main intermediate compounds during the degradation process (list of ions corresponding to each model pollutant can be found in the SI, table S1, S2 and S3).

To confirm some of the identifications made by LC-MS, aqueous $\mathrm{HNO}_{3}$ solutions $(\mathrm{pH}=3)$ of pure commercial products with concentrations ranging from $10^{-4}$ to $8 \times 10^{-4} \mathrm{~mol} \mathrm{~L}^{-1}$ were prepared. These solutions were injected in the chromatogram, with the same conditions as the corresponding degradation solutions. For each case, the retention time (RT) and the $\mathrm{m} / \mathrm{z}$ value of the base peak (BP) were recorded and compared with those obtained for the corresponding component in the degradation solution. The identification was considered confirmed when the same $\mathrm{m} / \mathrm{z}$ and a RT differing from less than $10 \%$ were found. This procedure was especially performed for the three parent compounds, and for the transformation products referred in the next sections as $\mathbf{3}, \mathbf{6}, \mathbf{1 3}, \mathbf{1 6}, \mathbf{1 9 a}, \mathbf{1 9 b}, \mathbf{2 2}, \mathbf{2 4 a}$, and 26.

\section{2-4. Qtof-HRMS analysis}


The supernatants recovered during the degradation process for each pollutant were directly infused in High Resolution Mass Spectrometry (HRMS). The MS1 analysis was operated in positive ion mode for MB and negative ion mode for PNP and MO using an MicrOTOF-Q II Mass Spectrometer (Bruker Daltonics, Wissembourg, France) with an electrospray ionization (ESI) source and an orthogonal accelerator, reflector and detector. The mass-detection range was set at $\mathrm{m} / \mathrm{z}=50-3000$. The ESI source parameters were as follows: drying gas $\left(\mathrm{N}_{2}\right)$ flow rate, $5.0 \mathrm{~L} \mathrm{~min}^{-1}$; drying gas temperature, $200{ }^{\circ} \mathrm{C}$; nebulizing gas pressure, $20 \mathrm{psi}$; capillary voltage, $4500 \mathrm{~V}$ for positive mode and $3500 \mathrm{~V}$ for negative mode. The collision energy and the pre-pulse storage were maintained at $4 \mathrm{eV}$ and $5 \mu \mathrm{s}$, respectively. For MS2 analysis, the targeted ion was selected in the quadrupole before its fragmentation with nitrogen in CID cell. The collision energy was adapted according to the $\mathrm{m} / \mathrm{z}$ of the parent ion. The mass detection range was set at $\mathrm{m} / \mathrm{z}=50-400$. All data acquisitions were controlled by the TOF Control software (Version 3.4, Bruker Daltonics).

\section{2-5. IC analysis}

The supernatants at $\mathrm{t}=24 \mathrm{~h}$ were analyzed by ion chromatography (Dionex ICS3000 apparatus) equipped with both CS16 cation-exchange and AS15 anion-exchange columns. After separation of the catalyst, $100 \mu \mathrm{L}$ of supernatant was taken and directly injected in the ion-exchange columns. The analyses were done at $\mathrm{T}=30^{\circ} \mathrm{C}$, with $30 \mathrm{mmol} \mathrm{L^{-1 }}$ methanesulfonic acid aqueous eluent solution at a flow rate of 1 $\mathrm{mL} / \mathrm{min}$, and $38 \mathrm{mmol} \mathrm{L} \mathrm{L}^{-1} \mathrm{KOH}$ aqueous eluent solution at a flow rate of $1.2 \mathrm{~mL} / \mathrm{min}$, respectively for cations and anions. The eluted ions were detected by conductimetry. They were identified by comparison of their retention time with that of the corresponding commercial products. Thus, aqueous $\mathrm{HNO}_{3}$ solutions $(\mathrm{pH}=3)$ of pure commercial products (referred in the next sections as $\mathbf{9}, \mathbf{1 0}, \mathbf{1 1}, \mathbf{1 2}, \mathbf{1 3}$, and 23) with concentrations ranging from $10^{-5}$ to $10^{-4} \mathrm{~mol} \mathrm{~L}^{-1}$ were prepared. These solutions were injected in the chromatogram, with the same conditions as the corresponding degradation solutions. Furthermore, Fo-to discriminate between the ions resulting from the degradation of the model pollutants 
and the trace ions resulting from the addition of $\mathrm{H}_{2} \mathrm{O}_{2}, \mathrm{HNO}_{3}$ or catalyst, a blank sample was analyzed by injecting the supernatant after $24 \mathrm{~h}$ of a catalytic test in the absence of the pollutant.

\section{Results and discussion}

\section{3-1. Synthesis and characterization of the catalyst:}

The synthesis and characterization of the $\gamma \mathrm{F}_{2} \mathrm{O}_{3} / \mathrm{SiO}_{2} \gamma-\mathrm{Fe}_{2} \mathrm{O}_{3} / \mathrm{SiO}_{2}$ catalyst have been thoroughly described in our previous paper [15]. In brief, the maghemite nanoparticles $\left(\gamma \mathrm{F}_{2} \mathrm{O}_{3} \gamma-\mathrm{Fe}_{2} \mathrm{O}_{3} \mathrm{NP}\right)$ were first prepared by co-precipitation followed by an oxidation step. Then, they were added to a silica precursor which was polymerized by sol-gel in a water-in-oil emulsion, leading to nanocomposite $\gamma$ $\mathrm{F}_{2} \mathrm{O}_{3} / \mathrm{SiO}_{2} \gamma-\mathrm{Fe}_{2} \mathrm{O}_{3} / \mathrm{SiO}_{2}$ microspheres [15,53]. Finally, the material was calcined at $400^{\circ} \mathrm{C}$ to remove any organic traces. This heterogeneous catalyst is characterized by a strong magnetic susceptibility, which is spectacularly confirmed by its behaviour in presence of a Nd-Fe-B magnet (see the photographs in fig. 1A). The $\gamma / \mathrm{F}_{2} \mathrm{O}_{3} / \mathrm{SiO} \mathrm{O}_{2} \gamma-\mathrm{Fe}_{2} \mathrm{O}_{3} / \mathrm{SiO}_{2}$ particles are strongly attracted by the magnetic force, which is proportional to the strong magnetic field gradient, and can be therefore separated from the aqueous solution in a very short time. This property considerably facilitated the analyses of the supernatants during the catalytic tests. The strong magnetic susceptibility of the catalyst, which results from the superparamagnetic behaviour of the $\gamma \mathrm{F}_{2} \mathrm{O}_{3} \quad \gamma-\mathrm{Fe}_{2} \mathrm{O}_{3} \quad \mathrm{NP}$, has been also confirmed by SQUID magnetometry [15].

The diffraction patterns (fig. 1B) obtained for the $\gamma-\mathrm{F}_{2} \mathrm{O}_{3} \gamma-\mathrm{Fe}_{2} \mathrm{O}_{3} \mathrm{NP}$ before and after their encapsulation into the silica beads are similar, and correspond well to the spinel structure of maghemite, confirming that the nanoparticles were not altered during the synthesis of the catalyst. The average crystal size of the nanoparticles calculated from the XRD patterns using the Scherrer formula is $d=7.1 \mathrm{~nm}$, which agrees well with the mean diameter of the $\gamma-\mathrm{Fe}_{2} \mathrm{O}_{3} \mathrm{NP}$ usually obtained by this method. The absence of modification of the $\gamma-\mathrm{Fe}_{2} \mathrm{O}_{3}$ NP inside the silica was also confirmed by UV-Vis-NIR spectroscopy [15].

The morphology and internal structure of the catalyst were examined by SEM and TEM (see fig. 1C and fig. 1D). The SEM micrograph of the material (Fig. 1C) indicated that the catalytic microspheres are 
polydisperse in size, with a relatively smooth external surface. Their mean diameter determined by counting the size of more than 350 beads is $\mathrm{D}=2.0 \mu \mathrm{m}$ with a standard deviation of $1.7 \mu \mathrm{m}$. The TEM micrograph of a bead (fig. 1D) showed a homogeneous and isotropic dispersion of the $\gamma-\mathrm{Fe}_{2} \mathrm{O}_{3} \mathrm{NP}$ inside the silica, without evidence for the formation of agglomerates, despite the relative high weight fraction of the $\gamma-\mathrm{Fe}_{2} \mathrm{O}_{3} \mathrm{NP}$ (a value of $8.1 \%$ w/w of $\gamma-\mathrm{Fe}_{2} \mathrm{O}_{3}$ in the silica was found by atomic absorption spectrophotometry).

The porosity of the material was evaluated by sorption volumetry of $\mathrm{N}_{2}$ and $\mathrm{CO}_{2}$ (respectively at $77 \mathrm{~K}$ and $273 \mathrm{~K}$ ) [15]. $\mathrm{N}_{2}$ volumetry have revealed that the microspheres are microporous, with pore diameters smaller than $2 \mathrm{~nm}$. A surface area of $744 \mathrm{~m}^{2} \mathrm{~g}^{-1}$ and a microporous volume of $0.30 \mathrm{~mL} \mathrm{~g}^{-1}$ have been found by $\mathrm{CO}_{2}$ adsorption volumetry [15]. These values confirm the large amount of micropores in the silica network. A mean diameter of $1.6 \mathrm{~nm}$ has been estimated for the micropores. This relatively large size is sufficient to allow the diffusion of the pollutants up to the catalytic sites located on the surface of the encapsulated $\gamma-\mathrm{Fe}_{2} \mathrm{O}_{3} \mathrm{NP}$.
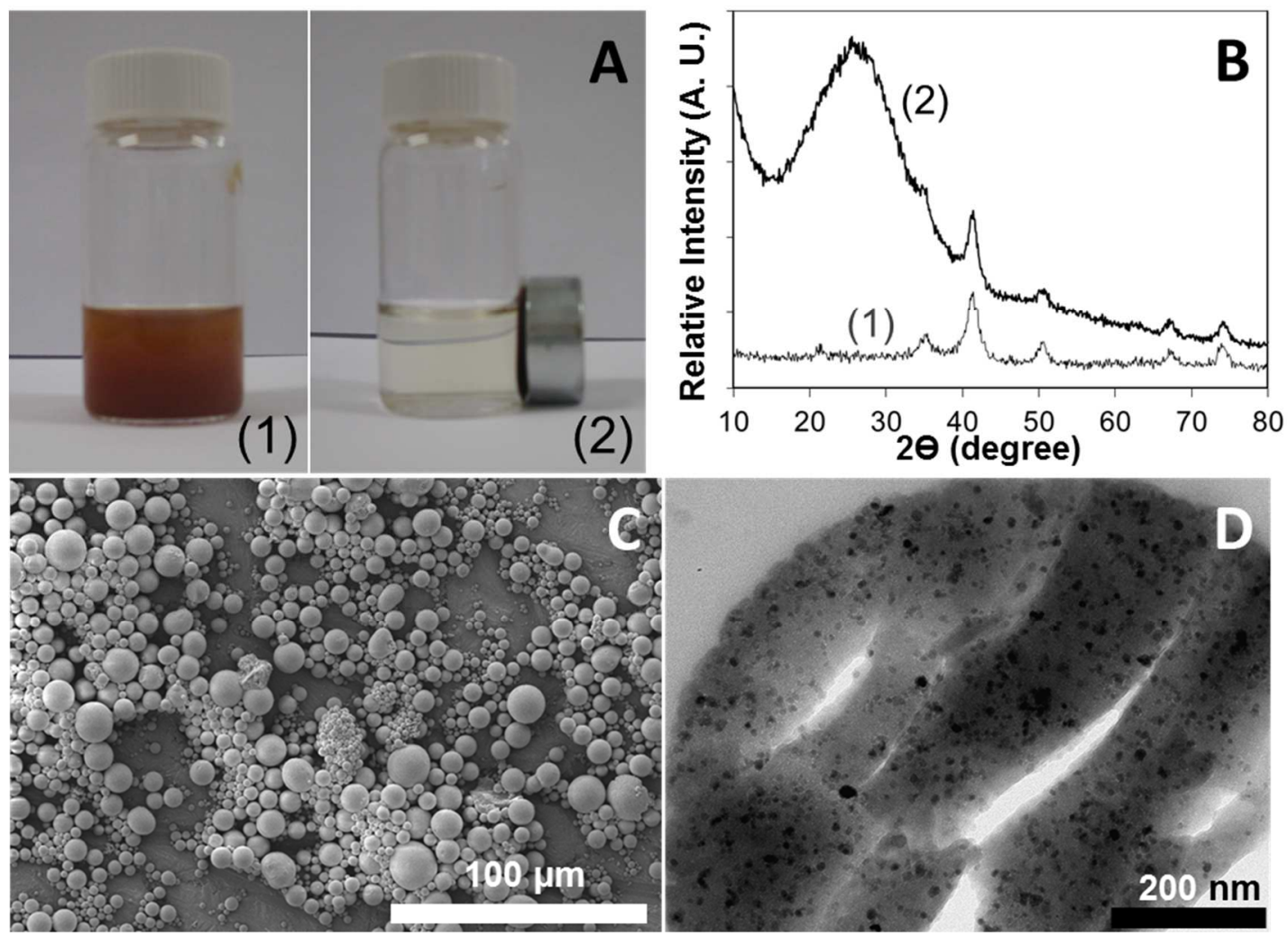

Figure 1: (A) Photographs of the water suspension of the $\gamma / \mathrm{F}_{2} \mathrm{O}_{3} / \mathrm{SiO} \mathrm{S}_{2} \gamma-\mathrm{Fe}_{2} \mathrm{O}_{3} / \mathrm{SiO}_{2}$ catalyst, without a magnet (1), and after two minutes in presence of a magnet (2); (B) XRD patterns of the $\gamma \mathrm{F}_{2} \Theta_{3} \gamma-\mathrm{Fe}_{2} \mathrm{O}_{3}$ 
NP before (1), and after (2), their encapsulation into the silica; (C) SEM image of the catalyst; (D) MET image of a single catalytic bead.

\section{3-2. Catalytic activity on the three pollutants}

The activity of the catalyst on the $\mathrm{H}_{2} \mathrm{O}_{2}$ oxidation of the three model aqueous pollutants, $\mathrm{MO}, \mathrm{MB}$ and PNP, have been studied for a large range of experimental conditions, as described in our previous work [15]. We have shown that the better catalytic activities are obtained at $\mathrm{pH}=3$ and $\mathrm{T}=40^{\circ} \mathrm{C}$. These conditions were used in the present publication for the study on the degradation mechanism of the three pollutants. We have also checked that the major part of the pollutants removal is effectively due to the heterogeneous catalyst by measuring the catalytic activity of the supernatant after a $1^{\text {st }}$ run, and by analysis of the iron concentration in solution after $4 \mathrm{~h}$ [15]. We thus have proved that the percentages of leached iron remain less than $0.4 \%$, demonstrating the good stability of the catalyst. The decolorization and the mineralization kinetics of the pollutant solutions were respectively determined by UV-visible spectroscopy, and measurement of the NPOC. Figure 2 displays the kinetic curves obtained for the decolorization (fig. 2A) and mineralization (fig. 2B) of the three pollutants.

The addition of the catalyst allows significant decolorizations of each pollutant solutions after few hours (see fig. 2A). These rapid decolorizations correspond to a fast oxidation of the pollutants into uncoloured species, due to the reactions with the $\mathrm{HO}^{\bullet}$ radicals, which result from the $\mathrm{H}_{2} \mathrm{O}_{2}$ decomposition at the surface of the $\gamma-\mathrm{Fe}_{2} \mathrm{O}_{3}$ nanoparticles. Best results are obtained with $\mathrm{MB}$, since quantitative decolorizations are attained after $3 \mathrm{~h}$. In comparison, the decolorization rate of the MO solution is slightly lower, whereas much lower activities are obtained for PNP. 

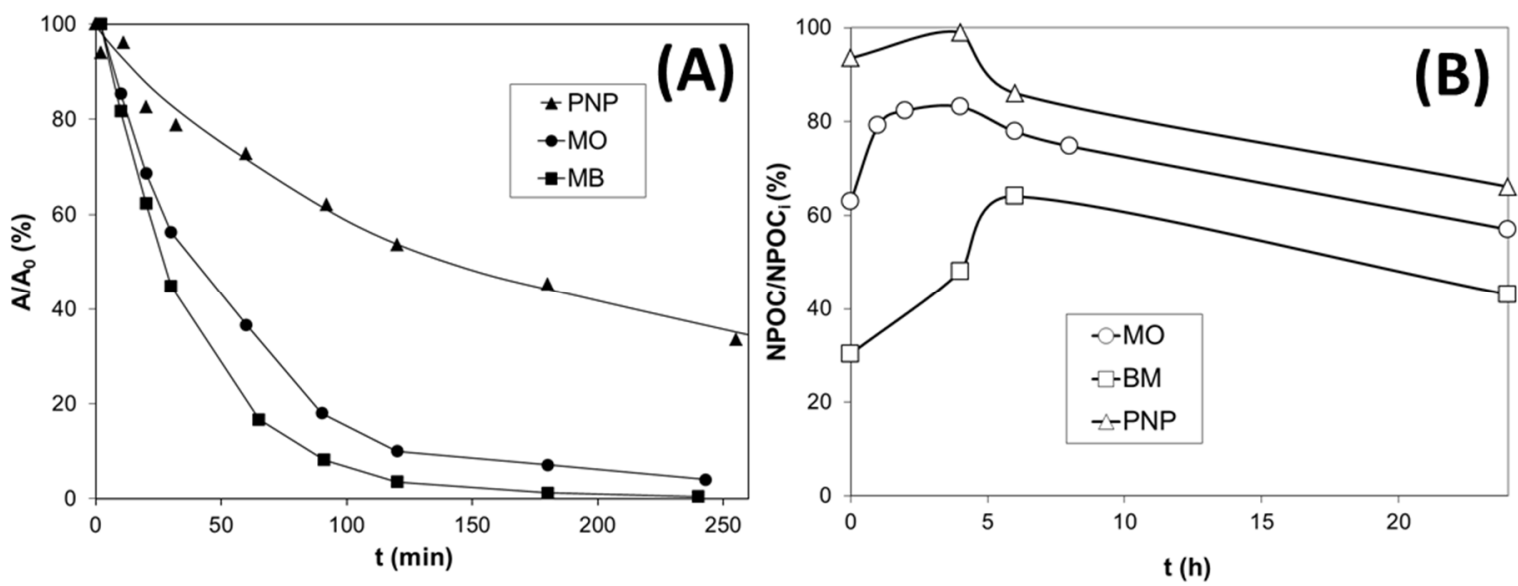

Figure 2: (A) Kinetic curves for the decolorization rates of the three pollutant solutions, $\mathrm{A} / \mathrm{A}_{0}$, where $\mathrm{A}$ and $\mathrm{A}_{0}$ are the absorbance at $\lambda_{\max }$ for each pollutant, respectively at $\mathrm{t}$ and $\mathrm{t}=0$; (B) Kinetic curves for the mineralization rates of the three pollutant solutions, NPOC/NPOC $i$, where $N P O C$ and $\mathrm{NPOC}_{\mathrm{i}}$ are the non-purgeable organic carbon in the supernatants, respectively at $\mathrm{t}$, and before adsorption equilibrium.

$$
\text { Squares }=\mathrm{MB} \text {; triangles }=\mathrm{PNP} \text {; circles }=\mathrm{MO}
$$

The mineralization of the pollutant solutions (fig. 2B) occurs much more slowly than their decolorization. Moreover, the patterns of the mineralization curves are rather complex. It should be noted, however, that the observed shapes are reproducible, although the relative uncertainties on the NPOC/NPOC $i$ values are relatively large $(+/-25 \%)$. At $\mathrm{t}=0$, the NPOC values are smaller than expected, because of the adsorption of a part of the pollutant on the catalyst (the $\mathrm{NPOC}_{\mathrm{NPOC}}$ at $\mathrm{t}=0$, corresponds to the maximum adsorption rate of the pollutant on the catalyst, which is of $70 \%, 37 \%$ and $7 \%$ for $\mathrm{MB}$, MO and PNP, respectively). During the first hours of the reaction, the amount of NPOC is increased, which can be interpreted in terms of desorption of the primary organic species resulting from the early degradation of the adsorbed pollutants. Finally, the NPOC is gradually decreased up to $24 \mathrm{~h}$, which is due to the slow mineralization of these primary organic species. Thus, for the three compounds, the real mineralization yields are relatively weak, and can be obtained by comparing the NPOC at the maximum of the kinetic curves with the NPOC at $24 \mathrm{~h}$. Thus, the real mineralization yields, which correspond to the oxidation of the pollutants into $\mathrm{CO}_{2}, \mathrm{H}_{2} \mathrm{O}$ and inorganic ions, range between 20 and $35 \%$.

It can be seen that there is a strong difference of reactivity between the pollutants regarding the decolorization rates, which correspond to the first steps of the oxidation process. This difference can be 
correlated to the adsorption rates. Indeed the same order is observed for the decolorization rates (see the kinetic curves in fig. 2A), and the initial amounts of adsorbed pollutant (see the NPOC/NPOC $\mathrm{i}_{\mathrm{i}}$ alues at $\mathrm{t}=0$ in fig. 2B). The correlation exiting between the adsorption and decolorization rates has been also mentioned in a recent paper, in which it has been evidenced that the reactivity of a pollutant toward an AOP can dramatically change, depending on its interaction with the catalyst support [54]. Therefore, a larger adsorption of a pollutant on the surface of the catalyst seems to favour a better catalytic activity, although the decolorization rate may be also influenced by other parameters such as the stability of the pollutant to the attack of the $\mathrm{HO}^{\circ}$ radicals, and the possible existence at the first stages of the process, of several transformation products having a similar coloration to-as the parent compound. The difference observed at early stages of the oxidation seems to have a poor influence on the mineralization rate, since the transformation into inorganic species occur very slowly from the ultimate organic intermediates, characterized by a reactivity toward $\mathrm{HO}^{\bullet}$ very different from the parent compound. Slow mineralization rates have also been reported for other AOPs, which have been explained by the accumulation of endproducts recalcitrant to mineralization $[22,23,26,31,42,45]$.

\section{3-3. Identification of the intermediate compounds and description of the degradation pathway-pathways}

$$
\text { 3-3-1 Methyl orange (MO) }
$$

\section{3-3-1-1 Identification of the intermediate compounds}

To assess the main degradation compounds corresponding to the MO oxidation, the supernatant was first analysed-analyzed by full-scan LC-MS at different times of catalysis $(\mathrm{t}=2 \mathrm{~min}, 30 \mathrm{~min}, 1 \mathrm{~h}, 2 \mathrm{~h}, 4 \mathrm{~h}$ and

24h). The chromatograms are given in figure 3. All the significant peaks are labeled with their retention time (RT) and the m/z value of highest ion in the corresponding MS spectrum (named base peak, BP). 
At short reaction times $(\mathrm{t}=2 \mathrm{~min}, 30 \mathrm{~min}$ and $1 \mathrm{~h}$ ), the chromatograms display a peak at $\mathrm{RT}=16.5 \mathrm{~min}$, with a BP at $\mathrm{m} / \mathrm{z}=304$. This peak is also found in the LC-MS chromatogram of the pure MO solution (not shown) and corresponds to $\mathrm{MO}\left(\mathrm{M}=304.3 \mathrm{~g} \mathrm{~mol}^{-1}\right)$. The area of this peak decreases as the reaction time increases, up to a complete disappearance after $1 \mathrm{~h}$ of reaction. This confirms that MO is totally oxidized during the reaction. Five major additional peaks are observed at short reaction times. The compounds associated to these peaks have been identified (see fig. 6 for their chemical structure), on the basis of the former studies on MO degradation [24,28-31,35,37,43,46,47]. The first peak at RT=1.6 min has been observed in all the chromatograms. It can be in fact decomposed in several poorly resolved peaks, which corresponds probably to several hydrophilic compounds, not well-separated in RPLC (based on hydrophobic interactions). However, the BP for this peak is at $\mathrm{m} / \mathrm{z}=320$, which corresponds to hydroxylation products of one of the two benzene rings of MO (compounds 1a). In addition, it should be noted the presence of increasing amounts of the ion at $\mathrm{m} / \mathrm{z}=336$, which may be ascribed to compounds resulting from a double hydroxylation of the benzene rings (compounds $\mathbf{5}$ ). The four other peaks at $\mathrm{RT}=$ 7.2, 11.2, 13.4 and $20.2 \mathrm{~min}$ can be attributed respectively to benzenesulfonate $(\mathrm{m} / \mathrm{z}=157$, compound 4), to a $\mathrm{N}$-demethylation product $(\mathrm{m} / \mathrm{z}=290$, compound 2 ) resulting from the cleavage of a $\mathrm{N}-\mathrm{C}$ bond in the dimethylamino group $\left(\left(\mathrm{CH}_{3}\right)_{2} \mathrm{~N}\right)$, to a species presenting both $\mathrm{N}$-demethylation and ring-hydroxylation $(\mathrm{m} / \mathrm{z}=306$, compound 4), and to an intermediate produced by ring-hydroxylation in ortho-position of the $\left(\mathrm{CH}_{3}\right)_{2} \mathrm{~N}$ group $(\mathrm{m} / \mathrm{z}=320$, compound $\mathbf{1 b})$. Compounds $\mathbf{2 , 3}$ and $\mathbf{4}$ have a lower RT than MO, which is consistent with their higher polarity. To the contrary, compound $\mathbf{1 b}$ is characterized by a larger RT, despite its higher polarity. As already described [24,28,29], to explain this apparent anomaly, it should be taken into account the existence of an internal hydrogen bond between the $\left(\mathrm{CH}_{3}\right)_{2} \mathrm{~N}$ group and the $\mathrm{OH}$ group in ortho-position. This internal hydrogen bond increases the hydrophobicity of the molecule, which results in a larger RT. Similarly, to explain that compound 4 was more retained in RPLC than compound 2 , we propose that the peak at $\mathrm{m} / \mathrm{z}=306$ is due to an isomer bearing an $\mathrm{OH}$ group in orthoposition of the $\mathrm{CH}_{3} \mathrm{NH}$ group. The existence of two peaks corresponding to $\mathrm{m} / \mathrm{z}=320$ ( $\mathrm{RT}=1.6 \mathrm{~min}$ and $\mathrm{RT}=20.2 \mathrm{~min}$ ), demonstrates the presence of at least two monohydroxylated isomers, characterized by a very different behaviors in terms of interactions with the LC column. To the contrary of the hydrophobic 
isomer at RT=20.2 min, it was not possible to precisely identify the hydrophilic isomer(s) at RT=1.6 min, since three structures can be associated to this peak, depending on the position of the $\mathrm{OH}$ group on the two benzene rings. Our results are in accordance with those of Dai et al., which have also noted the existence of several isomers resulting from ring-hydroxylation during the photocatalytic decomposition of MO [28]. At larger reaction time $(\mathrm{t}=2 \mathrm{~h}$ and $4 \mathrm{~h})$, the chromatograms are quite more complex with the gradual disappearance of the products observed at short reaction time, and the appearance of several small peaks, between $\mathrm{RT}=4.5 \mathrm{~min}$ and $6 \mathrm{~min}$, in which the ions at $\mathrm{m} / \mathrm{z}=173$ and 189 can be especially detected, and at $\mathrm{RT}=8.8 \mathrm{~min}$ where the presence of the ion at $\mathrm{m} / \mathrm{z}=276$ is attested. Finally, the chromatogram at very long reaction time $(\mathrm{t}=24 \mathrm{~h})$ exhibit exhibits only a single peak at $\mathrm{RT}=1.6 \mathrm{~min}$, without any dominating ion in the corresponding MS spectrum. This is indicative of the incapacity for RPLC (based on hydrophobic interactions) to separate the very hydrophilic compounds and/or small molecules produced at the end of the reaction. To complete the characterization of the final compounds, the sample at $\mathrm{t}=24 \mathrm{~h}$ was therefore analyzed by ion chromatography (IC). Several peaks were found in the anionic part of the chromatogram (see fig. S1, in the SI), especially at RT=3.9, 7.9, 9.2, 10.3 min and 16.9 min. By comparing these RT with those of the corresponding commercial products, these peaks were respectively assigned to formic acid, malonic acid, sulfate ions, oxalic acid, and nitrate ions (compounds $11,13,9,12$ and 10 ). Thus, the supernatant at $\mathrm{t}=24 \mathrm{~h}$ essentially contains small carboxylic acids and inorganic salts, which is in agreement with the former studies on the decomposition of azo dyes by AOP [26]. 


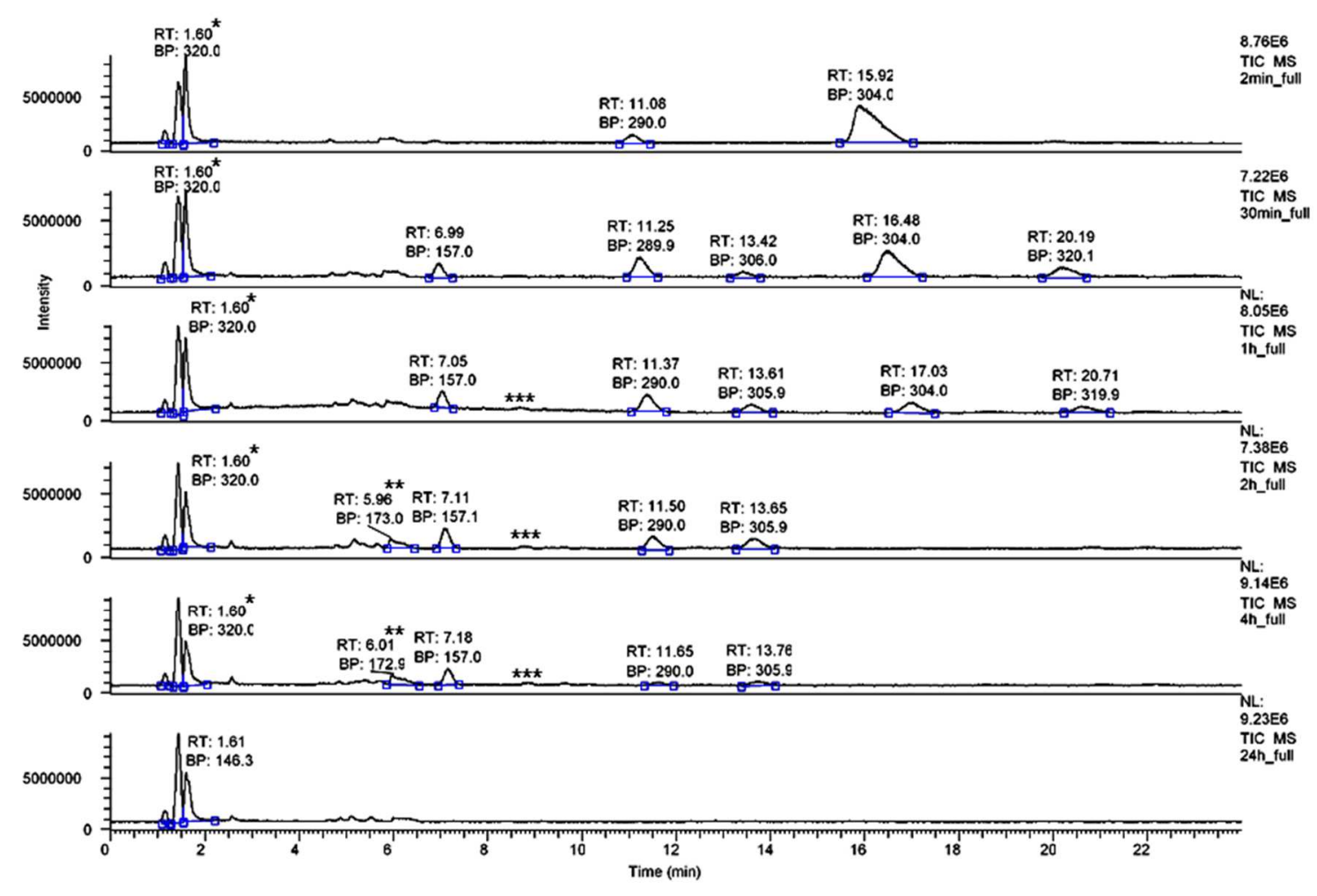

Figure 3: LC-MS chromatograms in full-scan mode corresponding to degradation of MO at different times ( $\mathrm{t}=2 \mathrm{~min}, 30 \mathrm{~min}, 1 \mathrm{~h}, 2 \mathrm{~h}, 4 \mathrm{~h}$ and $24 \mathrm{~h}$ ). Each chromatogram peak is characterized by its retention time (RT) and by the $\mathrm{m} / \mathrm{z}$ value of the highest ion in the corresponding mass spectrum (BP).

* : a substantial amount of the ion at $\mathrm{m} / \mathrm{z}=336$ is also found in SIM mode at $\mathrm{RT}=1.6$ min. ** : a substantial amount of the ion at $\mathrm{m} / \mathrm{z}=189$ is also found in the mass spectrum at $\mathrm{RT}=5.8 \mathrm{~min}$. $* * *$ : traces of the ion at $\mathrm{m} / \mathrm{z}=276$ are found in SIM mode at $\mathrm{RT}=8.8 \mathrm{~min}$.

To complete the identification of the MO degradation compounds, the samples corresponding to reaction times at $\mathrm{t}=30 \mathrm{~min}, 1 \mathrm{~h}$ and $4 \mathrm{~h}$ were analyzed by direct infusion in Qtof mass spectrometer. Figure 4 shows the MS1 spectrum and the MS2 spectra corresponding to the fragmentation of ion at $\mathrm{m} / \mathrm{z}=157$, 173, 290, 320 and 336, obtained after 4h of degradation. 

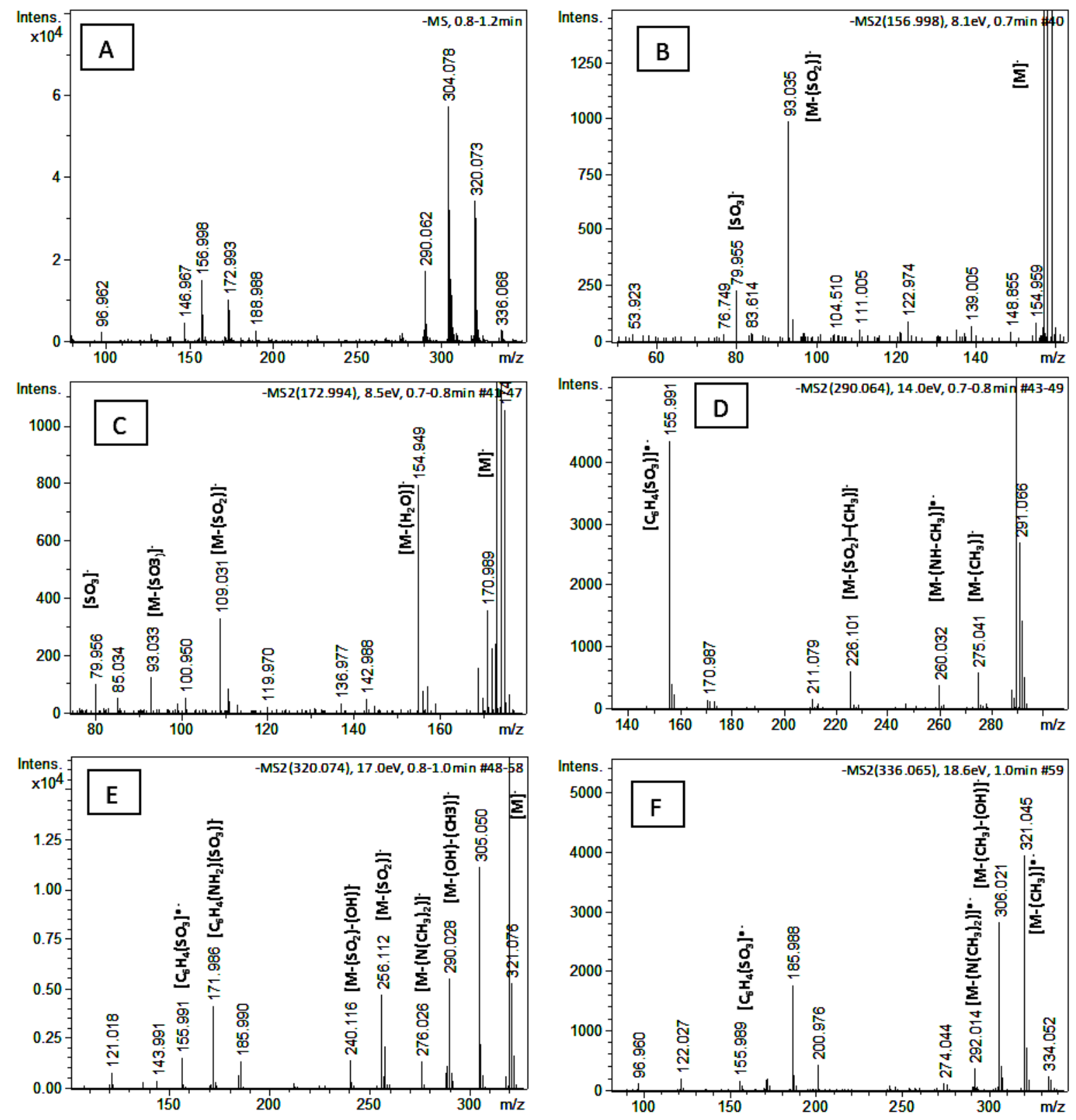

Figure 4: Qtof-HRMS analysis of supernatant of the MO solutions degraded at $t=4 \mathrm{~h}(\mathrm{~A})$ Mass spectrum in MS1 ; (B-F) MS2 spectra corresponding respectively to the parents ions: $\mathrm{m} / \mathrm{z}=156.998$ (B), 172.993 (C), 290.063 (D), 320.073 (E), 336.067 (F). When the fragments were identified, they were annotated in MS2 spectra

The MS1 spectrum (fig. 4(A)) exhibit exhibits several ions of high intensity, particularly at $\mathrm{m} / \mathrm{z}=$ $156.998,172.993,188.988,290.062,304.078,320.073$, and 336.068. These values are identical to the BPs of the main peaks in the LC-MS chromatograms (fig. 3) and can be therefore assigned to the molecular ions of the different transformation products. The exact masses of the MS1 molecular ions, and their corresponding fragment ions in the MS2 spectra were used, and the fragment ions were 
annotated in accordance with the fragmentation pattern described by Baiocchi et al. [24], to propose with high confidence (error <10 ppm) an elemental composition for each ion present on MS1 or MS2 spectra. Thus, the presence of benzenesulfonate as major degradation product is confirmed by the MS1 ion at 156.998 which corresponds with a high accuracy to the molecular formula $\mathrm{C}_{6} \mathrm{H}_{5} \mathrm{O}_{3} \mathrm{~S}$, and by the two fragment ions at $\mathrm{m} / \mathrm{z}=93.035$ and $\mathrm{m} / \mathrm{z}=79.955$ in the corresponding MS2 spectrum (fig. 4(B)), respectively attributable to the loss of $\mathrm{SO}_{2}$ and $\mathrm{C}_{6} \mathrm{H}_{5}$ groups. The presence of the $\mathrm{N}$-demethylated product is supported by the existence of the MS1 ion at $\mathrm{m} / \mathrm{z}=290.062\left(\mathrm{C}_{13} \mathrm{H}_{12} \mathrm{~N}_{3} \mathrm{O}_{3} \mathrm{~S}\right)$ and the MS2 fragment ions (fig. 4(D)) at $\mathrm{m} / \mathrm{z}=275.041,260.032,226.101,155.991$ (respectively, loss of $\mathrm{CH}_{3}$, $\mathrm{NH}\left(\mathrm{CH}_{3}\right), \mathrm{SO}_{2}$, and $\left.\mathrm{N}_{2} \mathrm{C}_{6} \mathrm{H}_{4} \mathrm{~N}\left(\mathrm{CH}_{3}\right)_{2}\right)$. In the same way, the existence of the ring-monohydroxylation products is confirmed based on the MS1 ion at $\mathrm{m} / \mathrm{z}=320.073\left(\mathrm{C}_{14} \mathrm{H}_{14} \mathrm{~N}_{3} \mathrm{O}_{4} \mathrm{~S}\right)$ and the corresponding MS2 fragment ions (fig.4(E)), while the ring-dihydroxylation products are inferred on the MS1 ion at $\mathrm{m} / \mathrm{z}=336.068,\left(\mathrm{C}_{14} \mathrm{H}_{14} \mathrm{~N}_{3} \mathrm{O}_{5} \mathrm{~S}\right)$ and the MS2 fragment ions (fig. 4(F)). Furthermore, the structure of the MS1 ion at $\mathrm{m} / \mathrm{z}=172.993\left(\mathrm{C}_{6} \mathrm{H}_{5} \mathrm{O}_{4} \mathrm{~S}\right)$, was elucidated as a ring-hydroxylated benzene sulfonate (compounds 7, fig. 4(C)). Although it was not possible to obtain the MS2 spectrum corresponding to the MS1 ion at $\mathrm{m} / \mathrm{z}=188.988$ because of its too low intensity, the presence of small amount of dihydroxylated benzene sulfonate (compounds 8) can be postulated, taking into account the good correlation between the observed and the theoretical $\mathrm{m} / \mathrm{z}$ corresponding to $\mathrm{C}_{6} \mathrm{H}_{5} \mathrm{O}_{5} \mathrm{~S}$. The existence of hydroxylated and dihydroxylated benzenesulfonates as transformation products in the course of the MO oxidation has been poorly documented, although it has been proposed in few studies $[30,37,43]$.

\section{3-3-1-2 Kinetics and degradation pathway for $M O$}

To have a better understanding of the MO degradation pathway, the evolution with time of degradation of the peak areas for the most abundant compounds identified detected in LC MS and identified thanks to HRMS was monitored by LC-MS in SIM mode. The results are depicted in figure 5. 


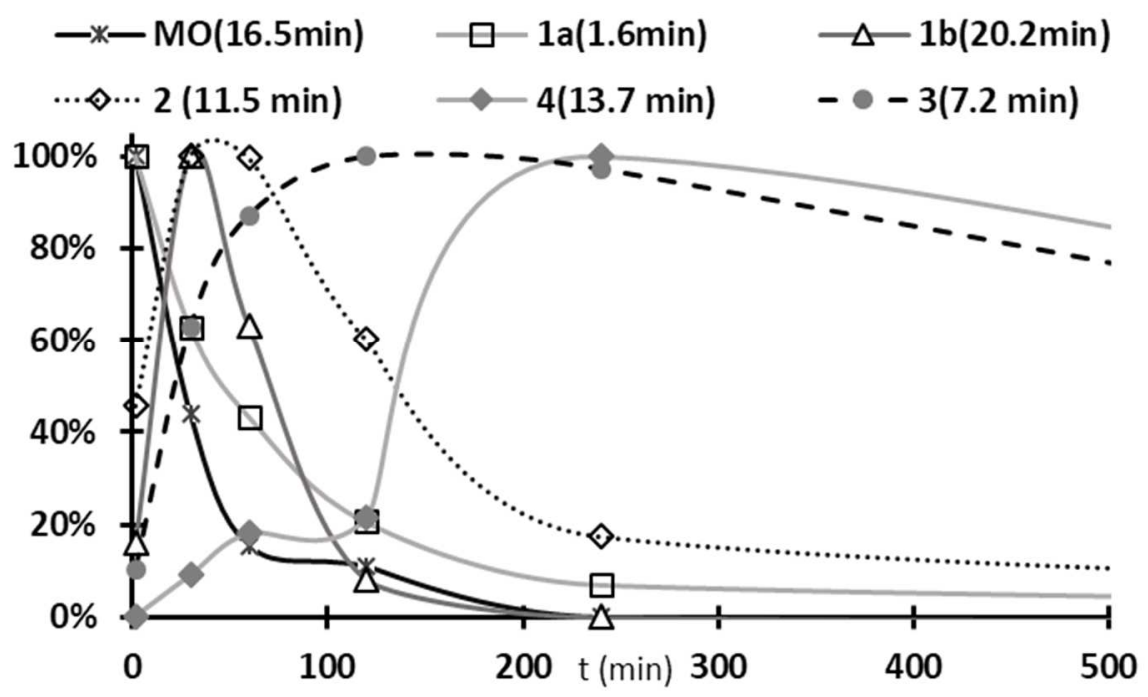

Figure 5 : Kinetics of the main intermediates identified for the MO degradation (evolution of the peak area determined during LC- MS analysis in SIM mode). Each peak area is normalized to $100 \%$ thanks to the maximum peak area reached for a given compound:

Several features can be observed from figure 5. First, a fast decrease of the peak area at $\mathrm{m} / \mathrm{z}=304$, $\mathrm{RT}=18.5 \mathrm{~min}$, is evidenced, confirming the fast and total oxidation of the parent compound, MO. At $\mathrm{t}=2$ min, several intermediates are already formed, especially those resulting from ring-hydroxylation (compound 1a and 1b), and N-demethylation (compound 2). These compounds can be considered as the first transformation products which results from the primary oxidation of MO. A fast decomposition of the hydroxylated isomers 1a occurs after $2 \mathrm{~min}$, indicating the lack of stability of this compound. The ortho-hydroxylated isomer 1b seems to have a better stability (with a maximum after 30 min of degradation). This higher stability may be due to the internal hydrogen bond between the $\mathrm{OH}$ and $\mathrm{N}\left(\mathrm{CH}_{3}\right)_{2}$ groups. Compound 2, has a maximum intensity after $60 \mathrm{~min}$, demonstrating that $\mathrm{N}$ demethylation takes longer than ring-hydroxylation, and/or that compound $\mathbf{2}$ has a better stability than compounds 1a and 1b. Compound $\mathbf{4}$ can be considered as a secondary intermediate, which results from the ortho-hydroxylation of compound $\mathbf{2}$, and/or from the N-demethylation of compound $\mathbf{1 b}$. Consequently, the maximum concentration for this transformation product is relatively late, with a maximum of presence after 4 hours of degradation. Benzenesulfonate (compound 3) results from the cleavage of the azo bond. This product is yet observed in very small amount at $\mathrm{t}=2 \mathrm{~min}$, indicating that it can be directly produced from MO. However, the maximum is found significantly later (at $\mathrm{t}=2 \mathrm{~h}$ ), which 
proves that this it is mainly formed from the first intermediates of the MO oxidation (compounds $\mathbf{1 a}, \mathbf{1 b}$ and 2). Moreover, the relatively slow decrease of the benzenesulfonate concentration after $2 \mathrm{~h}$ is indicative of its good stability toward oxidation, the most likely hypothesis being that it is slowly transformed into hydroxylated and dihydroxylated benzenesulfonates (compounds $\mathbf{7}$ and $\mathbf{8}$,) which were principally detected after $4 \mathrm{~h}$.

The overall proposed transformation pathway of MO during the Fenton oxidation in presence of the catalysts is given in figure 6. All the compounds displayed, and the methods used for their identification are also listed in table S1 of the SI.

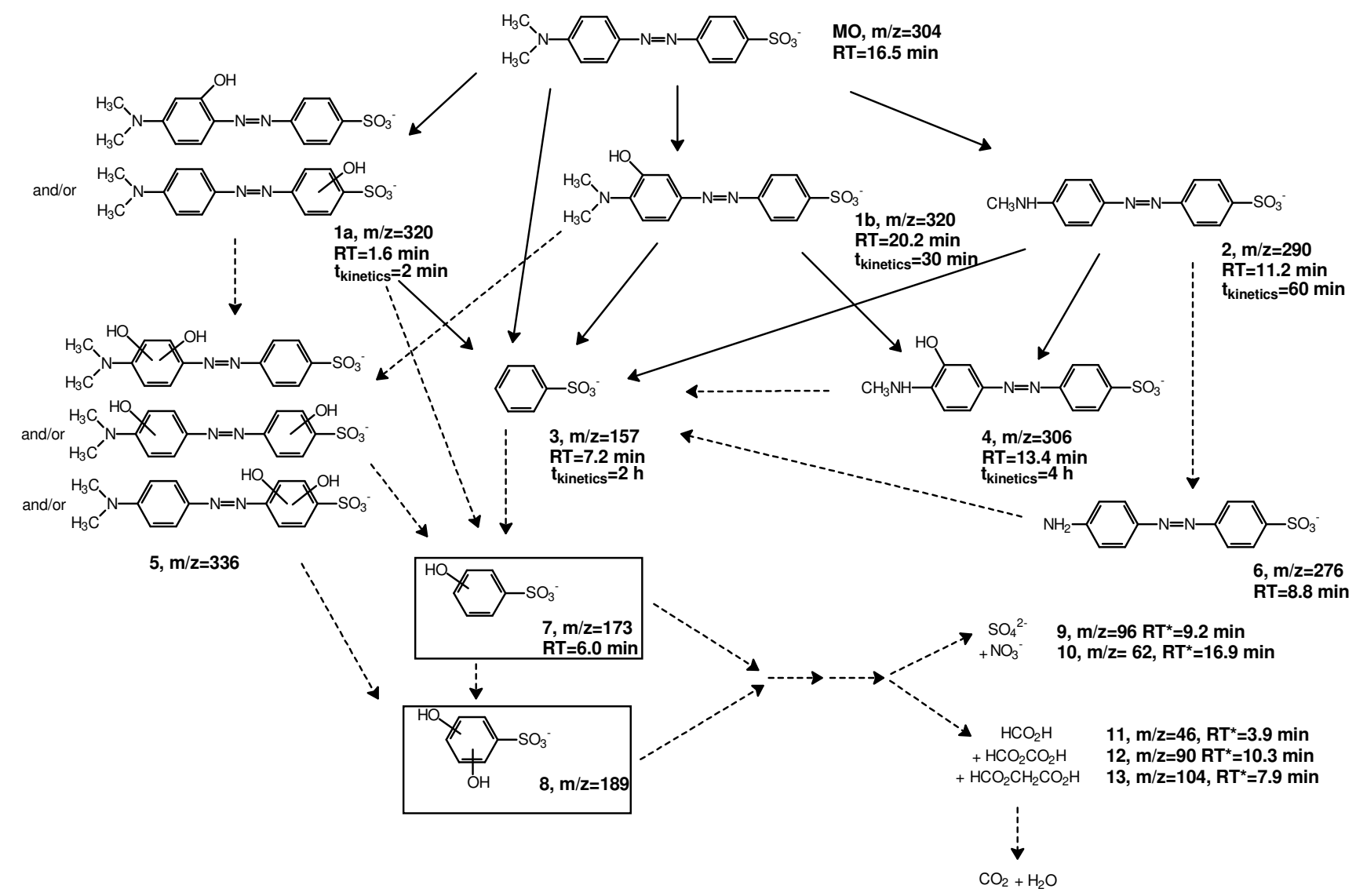

Figure 6 : Proposed degradation pathway for MO. The continuous arrows correspond to the confirmed steps of the MO degradation leading to the main intermediates, which evolution was monitored by LC-MS analysis in SIM mode. The dashed arrows correspond to possible steps leading to secondary or late intermediates. The framed compounds correspond to unusual intermediates with respect to previous studies. RT and $\mathrm{RT}^{*}$ are the retention times, respectively in full-scan LC-MS, and IC. $\mathrm{t}_{\text {kinetics }}$ corresponds to the time of degradation of MO leading to a maximum intensity for the given compound. 
The oxidation pathway of figure 6 results from the regioselectivity of the reactions with the $\mathrm{HO}^{\bullet}$ radicals produced at the surface of the catalyst. At least four primary intermediates can be formed, depending on the site of attack of the $\mathrm{HO}^{\bullet}$ radicals on the $\mathrm{MO}$ molecule. Compounds $\mathbf{1 a}$ and $\mathbf{1 b}$ result from the attack of the $\mathrm{HO}^{\bullet}$ radicals on one of the two the benzene rings, leading to a radical adduct which quickly evolves into a hydroxylated aromatic compound, according to reaction (1). Compound $\mathbf{1 b}$, results from an attack of the $\mathrm{HO}^{\circ}$ radicals on the benzene ring in ortho-position of the $\mathrm{N}\left(\mathrm{CH}_{3}\right)_{2}$ which is favored both by the strong electro-donating and the ortho-para directing effects of this group. Three isomers may correspond to compounds 1a. They result from ring-hydroxylation of the first benzene ring in metaposition of the $\mathrm{N}\left(\mathrm{CH}_{3}\right)_{2}$ group, and/or on the second benzene ring in ortho- or meta-position of the $\mathrm{SO}_{3}{ }^{-}$ group. The existence of these products has been explained by the strong reactivity of the $\mathrm{HO}^{\bullet}$ radicals which are able to overcome the ortho-para directing effects of the $\mathrm{N}\left(\mathrm{CH}_{3}\right)_{2}$ group, and/or the electronwithdrawing effect of the $\mathrm{SO}_{3}{ }^{-}$group [31].

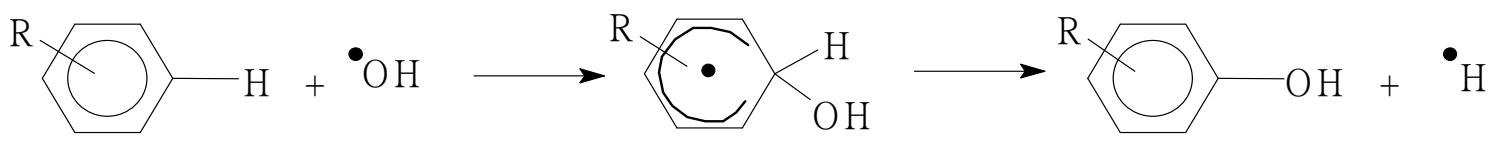

The presence of benzenesulfonate 3 at the first stages of the reaction is related to a reaction with a $\mathrm{HO}^{\circ}$ radical in the ipso positions of the azo group according to reaction (2). This leads to cleavage of the azo bond with the forming of two aromatic compounds, respectively bearing the sulfonate and the amino groups [55]. The existence of benzenesulfonate demonstrates that the main site of the ipso-attack is located on the benzene cycle bearing the dimethylamine, which is explained by the strong electrodonating ortho-para directing effect of this group. The lack of detection of the hydroxylated aromatic amine can be explained by its difficulty to be ionized in ESI(-) mode.

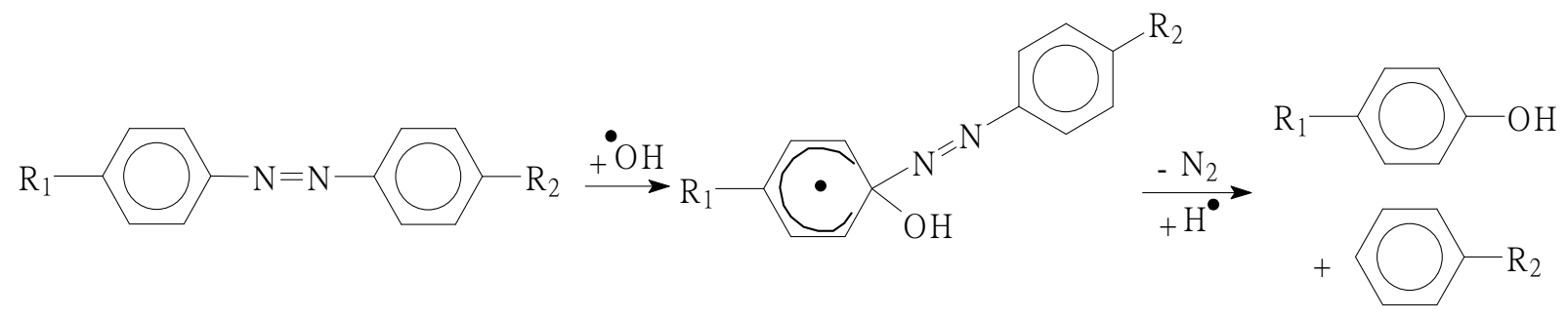


The last site for the attack of the $\mathrm{HO}^{\bullet}$ radicals is situated on the methyl group of the amino function. This proceeds by reaction (3) which is responsible for the formation of the N-demethylated compound 2. Demethylation of amine during the oxidation of azoic compounds has been frequently reported [24,26].

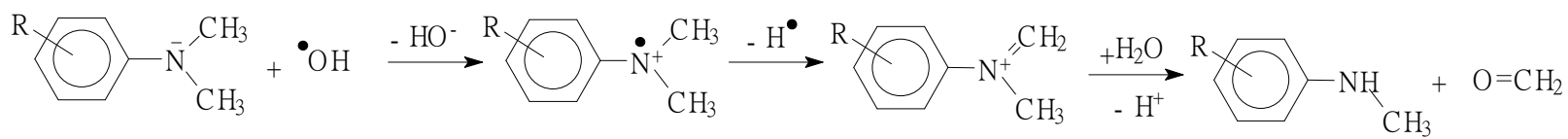

The overall degradation pathway of MO results from the combination of these three mechanisms (reactions (1)-(3)), which occurs successively and competitively. Our results obtained by LC-MS in SIM mode indicate that ring-hydroxylation (reaction (1)) is the main and the faster degradation pathway at the first stages, while demethylation (reaction (2)) may be considered as an important competitive mechanism. The presence of compounds $\mathbf{4 , 5}$ and $\mathbf{6}$ at later stages, indicates that successive reactions of $\mathrm{N}$-demethylation and ring-hydroxylation reaction continue to occur during several hours. Formation of benzenesulfonate 3 directly from MO, by cleavage of the azo bond, seems-is minor at the beginning of the oxidation process. Nevertheless, the presence of important amounts of this compounds and of its hydroxylated analogues (compounds 7-8) after several hours, while the concentration of demethylated and hydroxylated azo compounds has decreased, demonstrates that all the azo derivatives are slowly transformed into benzene sulfonates derivatives by cleavage of the azo bond (reaction (2)). The final disappearance of the benzenesulfonate analogues after $24 \mathrm{~h}$ of reaction, indicates that the ultimate steps of the oxidation process are ring-opening reactions which leads to inorganic salts (compounds $\mathbf{9}$ and 10), small carboxylic acids (compounds 11, 12 and 13), and $\mathrm{CO}_{2}$ and $\mathrm{H}_{2} \mathrm{O}$. Our results concerning the degradation of MO are globally in agreement with former studies on the degradation pathways of MO or similar azo dyes by other AOPs such as photocatalysis [24,26,28-31], or Fenton-type processes $[43,46,47]$. However we have highlighted the importance of the benzenesulfonate derivatives as late degradation intermediates.

\section{3-3-2 Methylene Blue (MB)}

\section{3-3-2-1 Identification of the intermediate compounds}

The main transformation products resulting from the MB Fenton oxidation have been similarly identified by LC-MS, Qtof-HRMS, and IC. The full-scan LC-MS chromatograms of the supernatants at 
different times of degradation (from $2 \mathrm{~min}$ to $24 \mathrm{~h}$ ) can be found in figure S2 (see SI). The peak at RT= 22 min with a $\mathrm{BP}$ at $\mathrm{m} / \mathrm{z}=284$ can be attributed to the parent compound $\mathrm{MB}$. This peak is no longer observed after two hours of reaction, confirming the total conversion of MB. The others peaks on the chromatograms eorrespends-correspond to the MB transformation products. The peaks at $\mathrm{RT}=21.7$, 21.2, 20.1, and $19.3 \mathrm{~min}$ with $\mathrm{BP}$ respectively at $\mathrm{m} / \mathrm{z}=270,256,256$ and 242 were easily identified through the former studies on MB degradation [22,23,27,38,40,44], and by comparing their RT with those of the corresponding commercial products. They have been attributed respectively to azure B (compound 16), the two isomers of azure A (compounds 19), and azure $C$ (compound 22). These compounds were formed by successive $\mathrm{N}$-demethylations from the $\mathrm{N}\left(\mathrm{CH}_{3}\right)_{2}$ groups of $\mathrm{MB}$. The peak at $\mathrm{RT}=23.8 \mathrm{~min}(\mathrm{BP}$ at $\mathrm{m} / \mathrm{z}=300)$ was supposed to be a hydroxylation product of $\mathrm{MB}$ (compound 15), while the peaks at $\mathrm{RT}=22.9$ and $23.3 \mathrm{~min}(\mathrm{BP}$ at $\mathrm{m} / \mathrm{z}=286)$ were considered to result from the combination of hydroxylation and N-demethylation (compounds $\mathbf{1 8}$ ). For MB, hydroxylation can occur on the aromatic rings or the sulphur atom. As with $\mathrm{MO}$, the high RT of these compounds stands for a ring-hydroxylation in ortho-position of the $\mathrm{N}\left(\mathrm{CH}_{3}\right)_{2}$ groups. It was more difficult to make an attribution for the other peaks on the chromatograms (BP at $\mathrm{m} / \mathrm{z}=217,231$ and 298). Qtof-HRMS was therefore carried out. Figure 7 shows the MS1 spectrum and the MS2 spectra corresponding to the ions at $\mathrm{m} / \mathrm{z}=217,231$ and 298, present after 2 hours of degradation. The MS1 spectrum (fig. 7(A)) exhibits several ions of high intensity (fig. S2) that can therefore be assigned to $\mathrm{MB}$ and its transformation products. The ions at $\mathrm{m} / \mathrm{z}=270.124,256.108,242.091$ and 300.128 enabled us to confirm the importance of the $\mathrm{N}$-demethylation and hydroxylation reactions. In addition, the presence of traces of the ion at $\mathrm{m} / \mathrm{z}=316.125$ (molecular formula $\mathrm{C}_{16} \mathrm{H}_{18} \mathrm{O}_{2} \mathrm{~N}_{3} \mathrm{~S}$ ) may also indicate the existence of dihydroxylation product(s) (compound(s) 17). The ion at $\mathrm{m} / \mathrm{z}=298.112$ matches well with the molecular formula $\mathrm{C}_{16} \mathrm{H}_{16} \mathrm{ON}_{3} \mathrm{~S}$. Thanks to its MS2 spectrum (fig. 7(B)), it was elucidated as being an aldehyde compound resulting from the oxidation of one of the methyl groups of MB (compound 14). The existence of aldehyde compounds in the course of MB oxidation has only been attested in few studies [22,38]. The ions at 231.075 and 217.059 in the MS1 spectrum correspond respectively to the molecular formulas $\mathrm{C}_{8} \mathrm{H}_{11} \mathrm{~N}_{2} \mathrm{O}_{4} \mathrm{~S}$ and $\mathrm{C}_{8} \mathrm{H}_{13} \mathrm{~N}_{2} \mathrm{O}_{3} \mathrm{~S}$. According to their fragmentation spectra (fig. 7(C-D)), we 
propose therefore to attribute these ions to small sulfonated molecules resulting from ring opening (compounds 20 and 21). This is the first time to the best of our knowledges that such compounds are observed in the course of MB degradation, although similar structures have been described by Houas $e t$ al. [22].
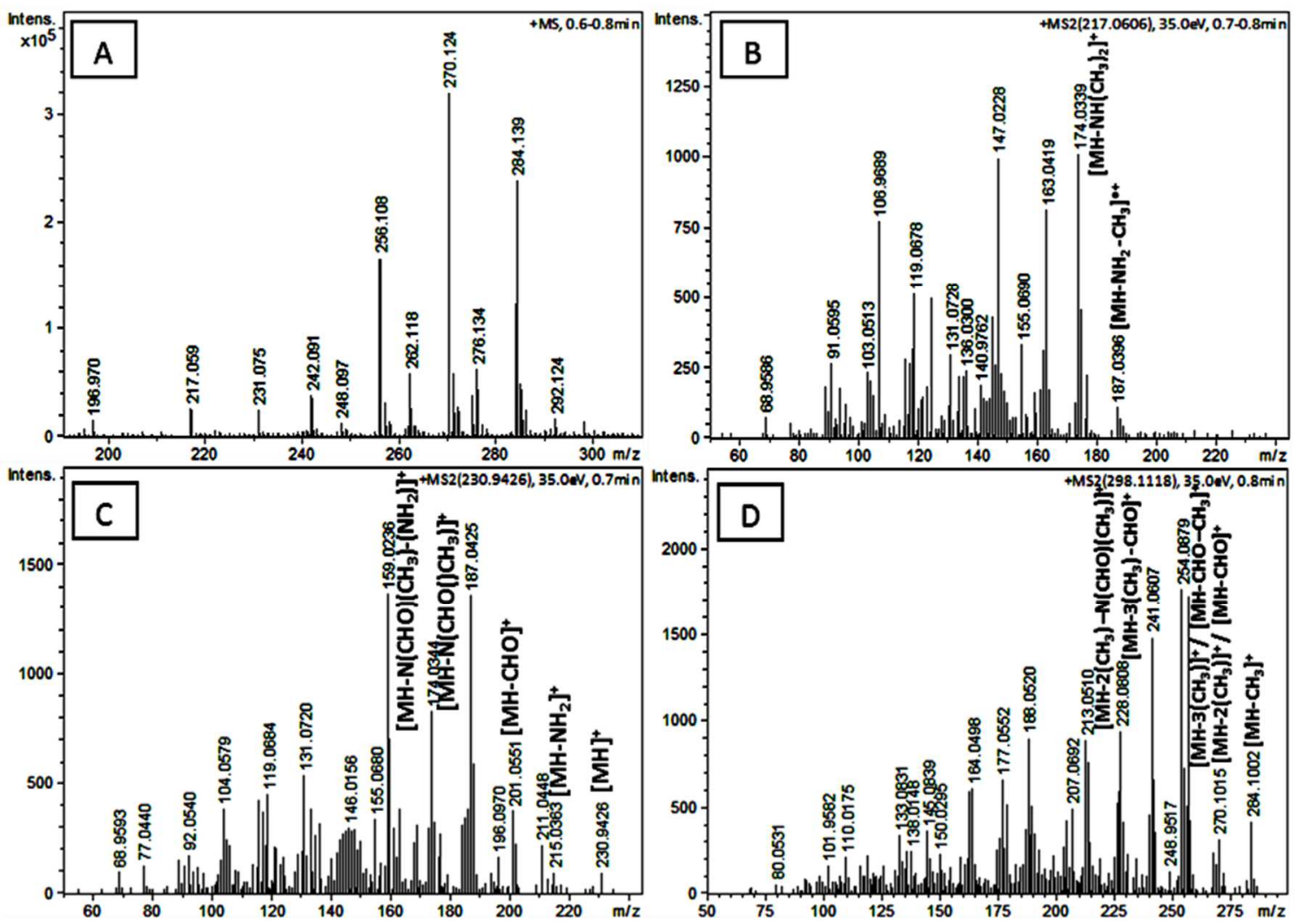

Figure 7: Qtof-HRMS analysis of supernatant of the MB solutions degraded at $t=2 \mathrm{~h}$. (A) Mass spectrum in MS1 ; (B-D) MS2 spectra corresponding respectively to the parents ions $\mathrm{m} / \mathrm{z}=217.0606$ (B), 230.9426 (C), 298.1118 (D).When the fragments were identified, they were annotated in MS2 spectra.

\section{3-3-2-2 Kinetics and degradation pathway for $M B$}

The evolution of the peak areas of the most abundant compounds detected during the course of MB degradation was evaluated by LC-MS in SIM mode (see figure S3 of the SI). All the intermediates are formed very early, with a maximum intensity after 2 or $30 \mathrm{~min}$ of degradation, after that their concentration progressively decreases up to a total disappearance after 2 hours. This indicates that during the first minutes, $\mathrm{MB}$ is partially decomposed by ring- or sulphur-hydroxylation, methyloxidation and $\mathrm{N}$-demethylation reactions into a first set of intermediates. These compounds and the 
remaining part of $\mathrm{MB}$ are then oxidized more slowly by other reactions. Among the second series of intermediates generated, the two isomers resulting from a second $\mathrm{N}$-demethylation are the main detected components, although small amounts of products resulting from a third N-demethylation (compound 22), or second hydroxylation (compounds 17), and two ring-opening products (compounds 20 and 21 ) are also formed. LC-MS was ineffective to identify the degradation compounds engendered at very late times ( $4 \mathrm{~h}$ to $24 \mathrm{~h}$ ). The sample at $24 \mathrm{~h}$ of degradation was therefore analyzed by IC (see fig. S1, in the SI). The peaks found were attributed to ethanoic acid, formic acid, malonic acid, sulfate ions, oxalic acid, and nitrate ions (compounds $23, \mathbf{1 1}, \mathbf{1 3}, \mathbf{9}, \mathbf{1 2}$ and 10), on the basis of the comparison with the RT of the commercial products. Thus, as for MO, the ultimate degradation compounds are composed of small mono- and di-carboxylic acids and inorganic salts such as sulfates and nitrates. The overall proposed transformation pathway for $\mathrm{MB}$ is given in figure 8 (see also table S2 in SI).

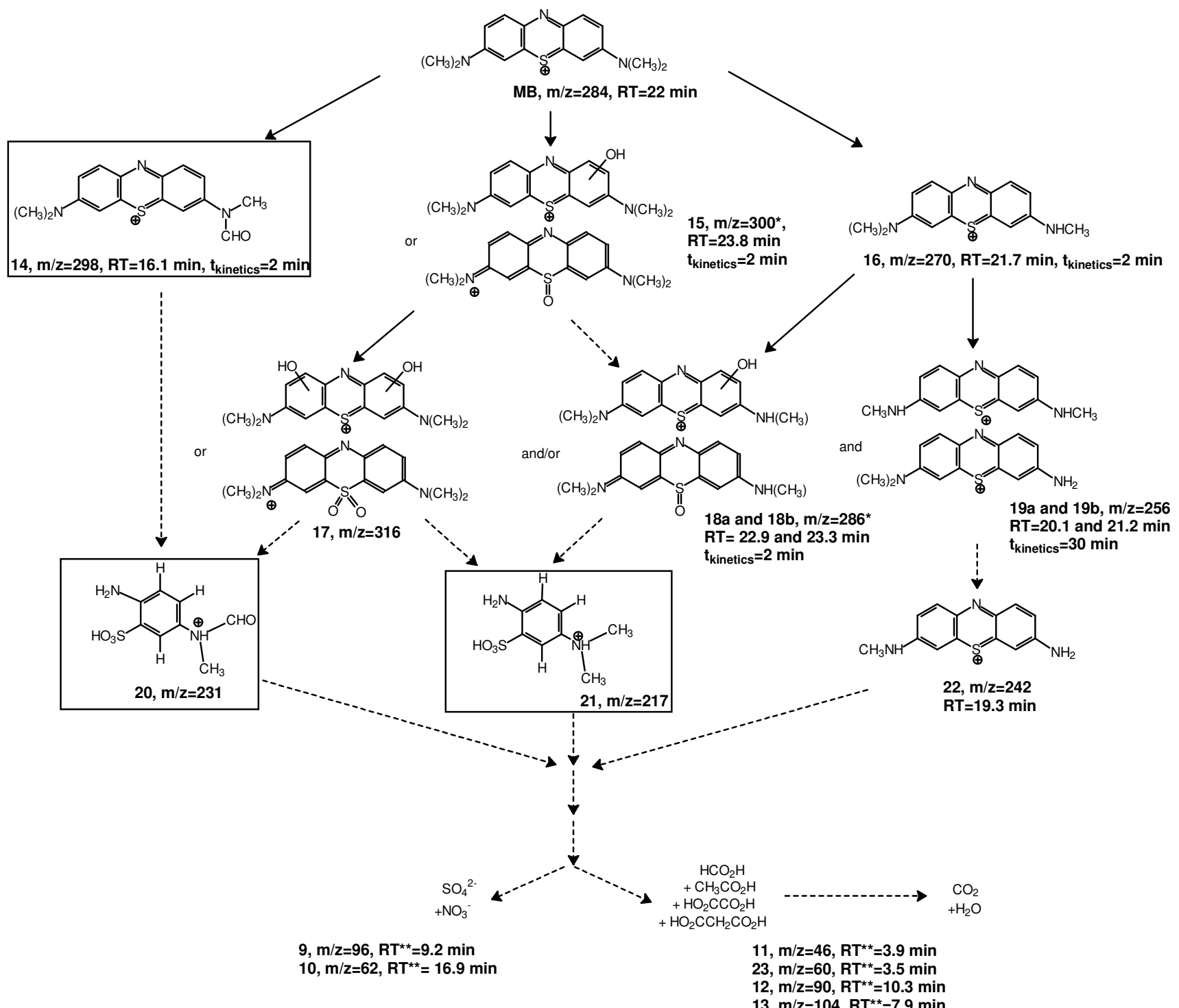

Figure 8: Proposed degradation pathway for MB. The continuous arrows correspond to the confirmed 
steps of the MB degradation leading to the main intermediates, which evolution was monitored by LCMS analysis in SIM mode. The dashed arrows correspond to possible steps leading to secondary or late intermediates. The framed compounds correspond to unusual intermediates with respect to previous studies. *= For $\mathrm{m} / \mathrm{z}=300$ and 286 , the compounds hydroxylated in ortho-positions of the amino groups are included in top structure which represents all the isomers hydroxylated on the aromatic rings; **=

\section{$\mathrm{RT}$ in IC.}

Figure 8 highlights the regioselectivity of the reaction between $\mathrm{MB}$ and the $\mathrm{HO}^{\bullet}$ radicals. Compound(s) 14 results from the attack of the $\mathrm{HO}^{\bullet}$ directly on the methyl group of the $\mathrm{N}\left(\mathrm{CH}_{3}\right)_{2}$ groups. This leads to a radical adduct which is rapidly transformed into an aldehyde function, according to reaction (4) [22]. The oxidation of the methyl group into an aldehyde function is also confirmed by the existence of the ring-opening product bearing an aldehyde function at $\mathrm{m} / \mathrm{z}=231$ (compound $\mathbf{2 0}$ ).

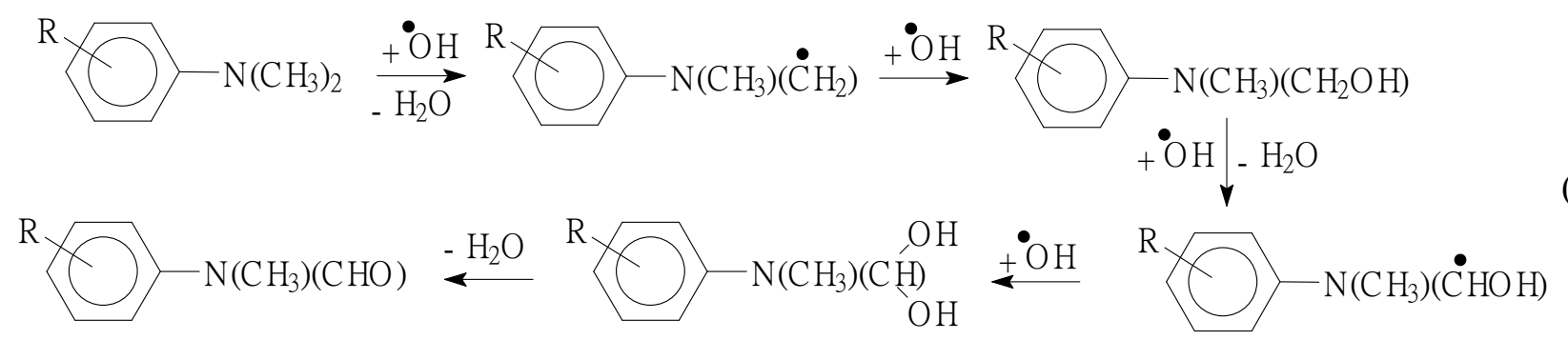

Compound(s) 15 results from a ring-hydroxylation mechanism similar to reaction (1), and/or from a direct oxidation of the sulphur atom into a sulfoxide function. Although the existence of sulfoxide derivatives as oxidation product of MB have been evidenced by several authors [22,27,38], it is more probable here that compound $\mathbf{1 5}$ corresponds to a ring-hydroxylation reaction occurring in ortho-position of the $\mathrm{N}\left(\mathrm{CH}_{3}\right)_{2}$ groups given its large RT in LC-MS. However small amounts of the sulfoxide derivative might also be formed, since traces of the ion at $\mathrm{m} / \mathrm{z}=300$ were also observed in LC-MS at lower RT. In addition, the existence of further oxygenation of the sulphur and aromatic rings is demonstrated by the presence of the dihydroxylated compound(s) at $\mathrm{m} / \mathrm{z}=316$ (compound(s) 17), and by the two ring-opening products (compounds $\mathbf{2 0}$ and $\mathbf{2 1}$ ) which result from the oxidation of the sulphur into a sulfonic acid.

Compound $\mathbf{1 6}$ is due to a $\mathrm{N}$-demethylation reaction, according to a mechanism similar to reaction (3). The high intensity of the ion at $\mathrm{m} / \mathrm{z}=270$ during the first 30 min indicates that compound $\mathbf{1 6}$ is the major 
secondary product directly generated from MB. The presence of appreciable amounts of the species resulting from consecutive $\mathrm{N}$-demethylations (compounds 19 and 22), confirms that these reactions are dominant during the first steps of the MB oxidation. However, ring- and sulphur-hydroxylation reactions probably play a key-role to explain the formation of ring-opening products, which leads finally to small organic acids and inorganic ions.

\section{3-3-3. Identification of the intermediate compounds and degradation pathway for paranitrophenol (PNP)}

The main decomposition products for the PNP Fenton oxidation have been identified by the same methods as MO and MB. The LC-MS chromatograms of the supernatants at different times of oxidation ( $\mathrm{t}=0 \mathrm{~min}, 2 \mathrm{~min}, 30 \mathrm{~min}, 1 \mathrm{~h}, 8 \mathrm{~h}$ and $24 \mathrm{~h}$ ) can be found in figure $\mathrm{S} 3$ (see $\mathrm{SI}$ ). The chromatograms present relatively few peaks, in comparison to $\mathrm{MO}$ and $\mathrm{MB}$. The peak at $\mathrm{RT}=21.3 \mathrm{~min}$ with a $\mathrm{BP}$ at $\mathrm{m} / \mathrm{z}=138$ was ascribed to the deprotonated form of the parent compound. The surface area of this peak progressively decreases, but to the contrary of $\mathrm{MO}$ and $\mathrm{MB}$, the conversion is total only after $8 \mathrm{~h}$, which confirms that the oxidation of PNP is relatively slow. Only two others peaks are observed in full-scan LC-MS. The first peak is found at RT=1.7 min and several ions of high intensity can be distinguished in the corresponding MS, especially at $\mathrm{m} / \mathrm{z}=139,155$ and 173 . These ions evolve differently with time, which indicates that several compounds are associated to this peak. The second peak is found at RT=4.2 min with a $\mathrm{BP}$ at $\mathrm{m} / \mathrm{z}=62$. It is also present in the chromatogram of a $10^{-3} \mathrm{~mol} / \mathrm{L} \mathrm{HNO}_{3}$ aqueous solution. It was then unambiguously attributed to the $\mathrm{NO}_{3}{ }^{-}$ions (compound 10).

A more sensitive method, based on SIM mode, was carried out to make the identification of the other degradation compounds for PNP. This enabled us to detect the ions at $\mathrm{m} / \mathrm{z}=103(\mathrm{RT}=3.5 \mathrm{~min}), 115$ $(\mathrm{RT}=8.1 \mathrm{~min})$, and $\mathrm{m} / \mathrm{z}=154(\mathrm{RT}=17.1,18.8$ and $22.7 \mathrm{~min})$. The ions at $\mathrm{m} / \mathrm{z}=103$ and 115 was assessed to the deprotonated forms of malonic and maleic acid (compounds $\mathbf{1 3}$ and 26), based on the injection of the corresponding commercial compounds. The three peaks corresponding to $\mathrm{m} / \mathrm{z}=154$ were supposed to be some isomers resulting from the ring-hydroxylation of PNP (compounds $24 \mathbf{a}, \mathbf{2 4 b}$ and $\mathbf{2 4 c}$ ). The peak at RT=17.2 min was attributed to 2-hydro-4-nitrophenol (compound 24a), through the injection of the 
corresponding commercial product. The attribution of the two other peaks was more difficult since several isomers may exist depending on the relative positions of the two hydroxyl and nitro substituents.

The obtained results have been completed by Qtof-HRMS. The MS1 spectrum of the sample at $\mathrm{t}=4 \mathrm{~h}$ exhibit exhibits several ions, especially at $\mathrm{m} / \mathrm{z}=88.988,103.003,115.004,138.020,154.015$, and 172.959 (fig. 9(A)). The ions at 115.004 and 103.003 confirm the presence of malonic and maleic acids (raw formula $\mathrm{C}_{4} \mathrm{H}_{3} \mathrm{O}_{4}$ and $\mathrm{C}_{3} \mathrm{H}_{3} \mathrm{O}_{4}$ ). The presence of PNP and hydroxylated-PNP is supported by the existence of the ions at 138.020 and 154.015 (raw formula $\mathrm{C}_{6} \mathrm{H}_{4} \mathrm{NO}_{3}$ and $\mathrm{C}_{6} \mathrm{H}_{4} \mathrm{NO}_{4}$ ) and by the fragmentations observed in the corresponding MS2 spectra (fig. 9(B) and 9(C)). The ion at $\mathrm{m} / \mathrm{z}=88.988$ was due to oxalic acid, the presence of this compound being also attested by IC. In addition, QtofHRMS enabled us to attribute the ion at $\mathrm{m} / \mathrm{z}=172.959$ to pentahydroxyphenol (compound 25), taking into account the good agreement with the raw formulae $\mathrm{C}_{6} \mathrm{H}_{5} \mathrm{O}_{6}$, and the MS2 spectrum (fig. 9(D)).
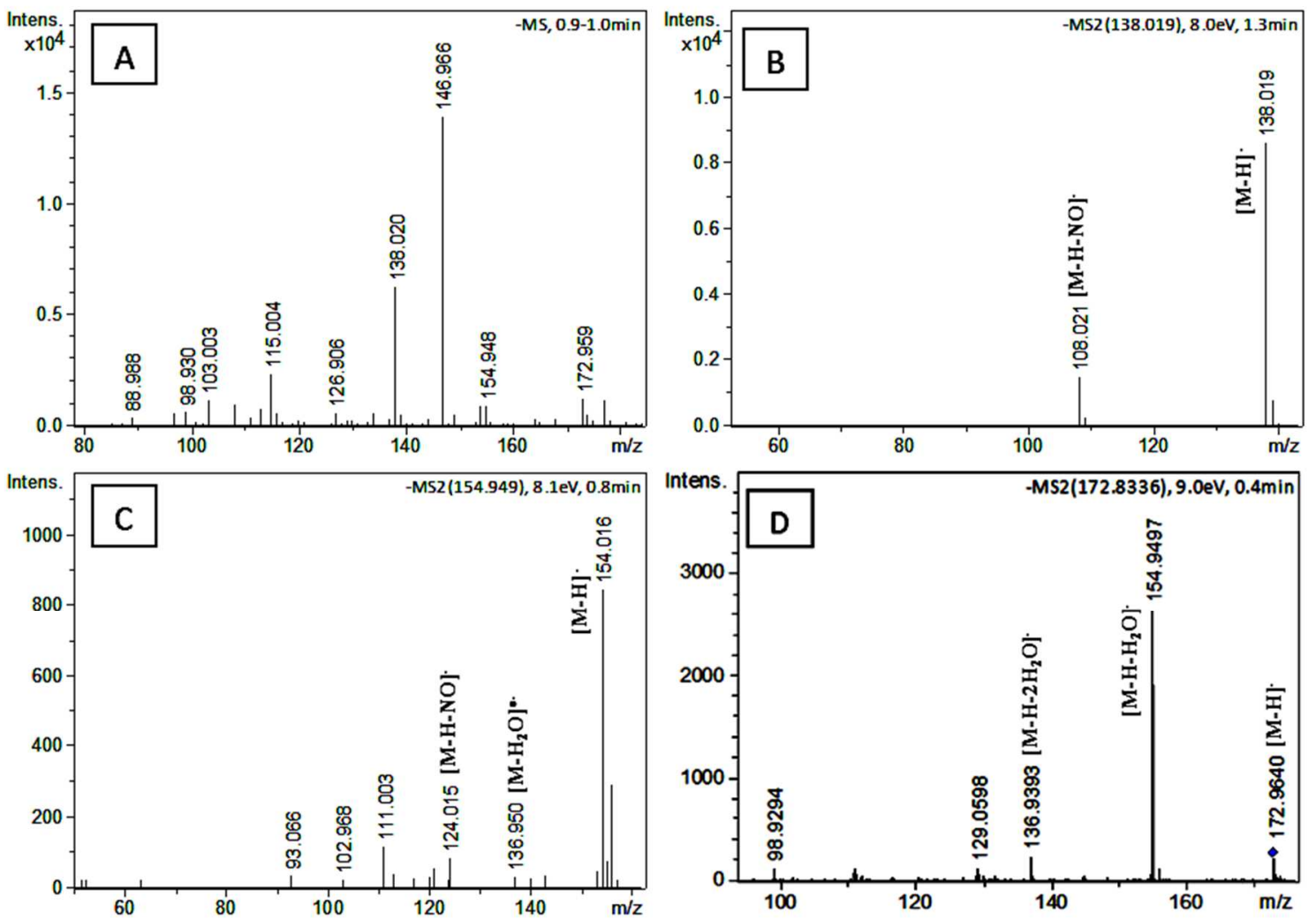

Figure 9: (A) Mass spectrum in MS1 Qtof corresponding to the supernatant of the PNP solutions degraded at $\mathrm{t}=240 \mathrm{~min}$; (B)-(D) Mass spectra in MS2 Qtof corresponding respectively to the parents ions: $\mathrm{m} / \mathrm{z}=138.019(\mathrm{~B}), 154.015(\mathrm{C})$, and 172.959. Some of the corresponding ions are indicated on the figures. 
Finally, the whole analyses performed for the oxidation of PNP enabled us to propose the overall transformation pathway given in figure 10 (see also table S3 in SI).

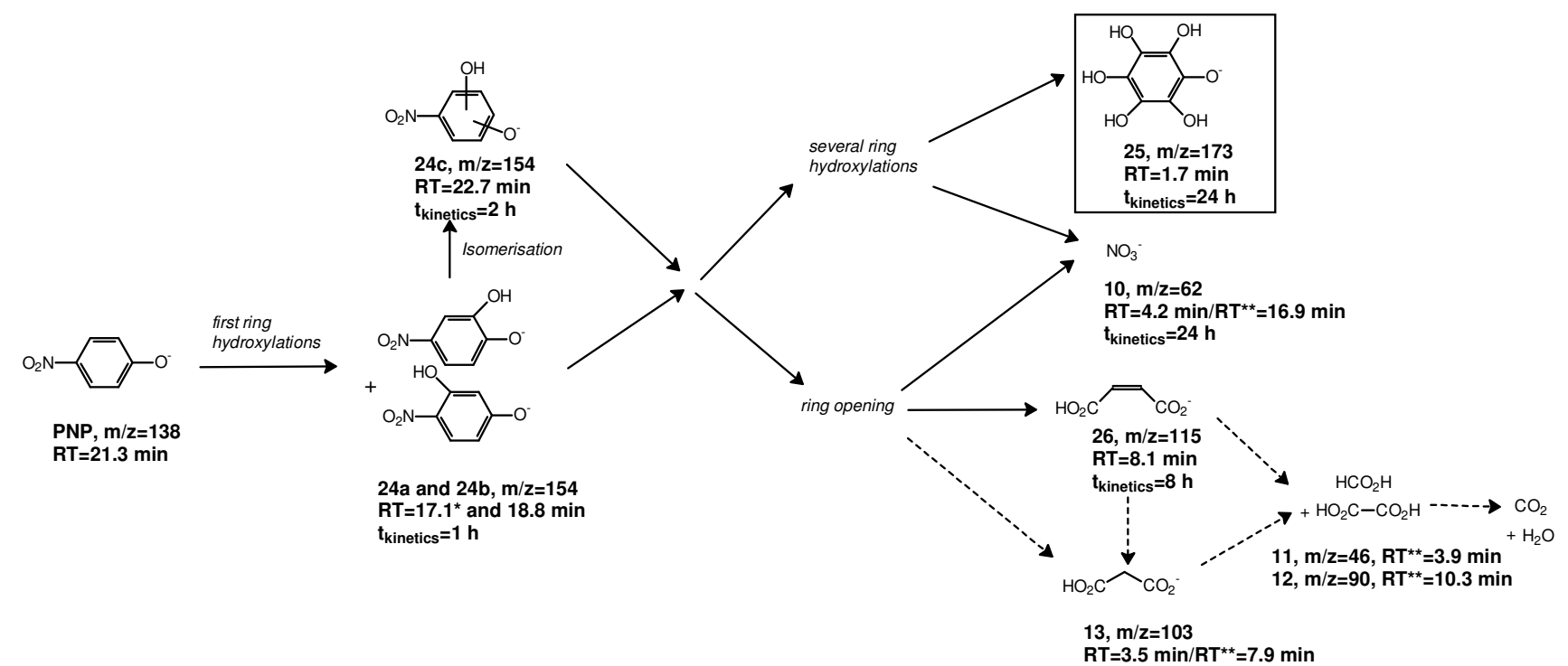

Figure 10 : Proposed degradation pathway for PNP. The continuous arrows correspond to the confirmed steps of the PNP degradation leading to the main intermediates, which evolution was monitored by LCMS analysis in SIM mode. The dashed arrows correspond to possible steps leading to secondary or late intermediates. The framed compounds correspond to unusual intermediates with respect to previous studies. *= 2-hydro-4-nitrophenol has been formally identified as the isomer corresponding to RT=17.2 $\min ; * *=$ RT in ion chromatography. All the $\mathrm{m} / \mathrm{z}$ values correspond to the deprotonated form $(\mathrm{M}-\mathrm{H})$ except for compounds 11 and $\mathbf{1 2 .}$

Our results prove that PNP is first oxidized by ring-hydroxylation (see reaction (1)). These reactions are dominant during the first $30 \mathrm{~min}$. Two isomers are formed, corresponding to compounds $\mathbf{2 4 a}$ and $\mathbf{2 4 b}$, respectively observed at $\mathrm{RT}=17.1$ and $18.8 \mathrm{~min}(\mathrm{~m} / \mathrm{z}=154)$. The major compound $24 \mathrm{a}$ has been clearly identified as 2-hydro-4-nitrophenol. It results from ring-hydroxylation in ortho-position of the phenolic group. We propose that the isomer $\mathbf{2 4 b}$ corresponds to the 3-hydro-4-nitrophenol, which results from hydroxylation in meta-position of the phenolic group. According to literature, ring-hydroxylations in ortho- and para-positions of the phenolic group are generally favoured, because of the strong electrodonating effect of this function $[21,25,32,45]$. For PNP, hydroxylation in para-position of the phenol group leads to the removal of the nitro substituent and to the formation of the 4-hydroxyphenol. Here, this compound has not been formally identified, although we have noticed traces of the ion at $\mathrm{m} / \mathrm{z}=109$ 
or 110 at $\mathrm{RT}=1.7 \mathrm{~min}$. Nevertheless, the presence of important amounts of the compound $\mathbf{2 5}$, which results from several successive ring-hydroxylations, and the continuous formation of $\mathrm{NO}_{3}^{-}$ion are consistent with the existence of the hydroxylation in para-position of the phenolic group. The difficulty to clearly identify 4-hydroxyphenol, and others by-products resulting from consecutive ringhydroxylations is due to the absence of separation of these very hydrophilic compounds on the LC column. A third hydroxylated isomer corresponding to $\mathrm{m} / \mathrm{z}=154$, at $\mathrm{RT}=22.7 \mathrm{~min}$ (compound $\mathbf{2 4 c}$ ) was tardily observed. Its concentration reaches a maximum at $\mathrm{t}=2 \mathrm{~h}$. It is therefore probable that this compound is formed by isomerization of the compounds $\mathbf{2 4 a}$ or $\mathbf{2 4 b}$. To explain its occurrence, it is important to note that the first step of the ring-hydroxylation (reaction (1)) produces dihydroxycyclohexadienyl radicals. These intermediates are known to give transposition reactions of the hydroxy substituents [56,57]. These transpositions may generate the positional isomer of hydroxynitrophenol corresponding to compound $\mathbf{2 4 c}$. The peak at $\mathrm{m} / \mathrm{z}=115$, identified as maleic acid (compound 26), is characterized by a maximum at $\mathrm{t}=2 \mathrm{~h}$. This ring-opening product is therefore produced by a consecutive reaction from the first oxidation products. It is important to note that compounds $\mathbf{2 6}$ is in turn oxidized, since its concentration ultimately decreases up to a total disappearance at $\mathrm{t}=24 \mathrm{~h}$. Three types of transformation products result from the late oxidation steps. The surface of the peak at $\mathrm{m} / \mathrm{z}=62$ progressively increases up to $\mathrm{t}=24 \mathrm{~h}$, showing that $\mathrm{NO}_{3}{ }^{-}$ions are continuously formed, by hydroxylation of the aromatic carbon bearing the nitro group. As for $\mathrm{MO}$ and $\mathrm{MB}$, the presence at $\mathrm{t}=24 \mathrm{~h}$ of small carboxylic acids such as malonic, formic and oxalic acids (compounds 13, 11 and 12) was detected by LC-MS and IC. Additionally, the presence at $\mathrm{t}=24 \mathrm{~h}$ of large amounts of the pentahydroxylated compound 25 suggests that the ring-opening transformations are slow. The existence of compound 25 has not yet been mentioned. Here, we have demonstrated that the remaining organic carbon at $24 \mathrm{~h}$ is partially due to polyhydroxylated phenols such as $\mathbf{2 5}$. This compound may be resistant to further oxidation leading to ring-opening and to mineralization. 


\section{Conclusion}

In this work, we have focused on the degradation pathway of three model aqueous pollutants, MO, MB and PNP, in a Fenton process based on the use of maghemite/silica microspheres as magnetically separable heterogeneous catalyst. Various transformation products were identified at different times, through the complementary utilization of different analytical methods, especially LC MS, HRMS (Qtøf in MS1 and MS2 mode) and IC. The kinetics of the main intermediate compounds was monitored by LC MS in SIM mode, which enabled us to propose an overall transformation pathway for each pollutant. Some of the intermediates have been already evidenced in other studies on the AOP degradation of these pollutants. However, we were able to identify new compounds, and to highlight some unusual decomposition steps. , particularly thanks to the highly accurate Qtof MS2 analyses.-The main advantage of our work is that a comparison between the three transformation pathways can be drawn.

Thus, aromatic ring-hydroxylation are observed for MO, MB and PNP. They display a certain regioselectivity, since they are observed preferentially in ortho- or para-position of the electrodonating groups such as $\mathrm{N}\left(\mathrm{CH}_{3}\right)_{2}$ or $\mathrm{OH}$. In addition, products resulting of several consecutive ringhydroxylations are systematically observed. For PNP, ring-hydroxylated compounds seems to be the unique transformation products at early time, while for $\mathrm{MB}$ and $\mathrm{MO}$ other products are competitively formed, especially those resulting from successive $\mathrm{N}$-demethylations of the $\left(\mathrm{CH}_{3}\right)_{3} \mathrm{~N}$ groups. For $\mathrm{MB}$, we have also noticed other types of competitive reactions, at the beginning of the process, especially the direct oxidation of the methyl groups into aldehyde. For the three compounds, products resulting from ring-opening and cleavages become appreciable only after several hours, although small amount of these products can be produced at the beginning of the process, especially for MO. Similar final oxidation products were detected. They are mainly composed of inorganics salts $\left(\mathrm{NO}_{3}^{-}, \mathrm{SO}_{4}{ }^{2-}\right)$, and small mono- or dicarboxylic acids such as formic, acetic, oxalic and malonic acids. These compounds have been described to be the ultimate oxidation products in many studies. They are very slowly transformed into $\mathrm{CO}_{2}$ and $\mathrm{H}_{2} \mathrm{O}$, which explain the relative low mineralization-mineralizations obtained at $24 \mathrm{~h}$. It is important to mention that for PNP, we have also highlighted the presence at $24 \mathrm{~h}$ of important amount of a polyhydrolylated phenolic compound. 
The differences between the three degradation pathways are explained by the differences in the structure of the parent compounds, which has an impact on the possible sites of reaction with the $\mathrm{HO}^{\bullet}$ radicals. The regioselectivity of the oxidation steps may be also influenced by the adsorption properties of the parent compound on the catalyst. While some common features with other advanced oxidations processes were found, it is difficult to conclude either the specificities are related to the conditions used here, or can be explained by the selected analytical methods. A partial answer may be given by studying the degradation pathway of the three pollutants in presence of more efficient heterogeneous catalysts, or by using visible light to improve the oxidation rates. Additional analytical methods (NMR, GC-MS...) may be also carried out to complete the identification of the transformation products.

\section{Acknowledgments}

We wish to kindly thank Emmanuel Aubry, Isabelle Pellerin and Isabelle Correia for their technical support, Anne Davidson, Olivier Lequin and Mohamed Salah Medjram for the fruitfull-fruitful discussions. We equally acknowledge the scientific cooperation research programme-program PROFAS between France and Algeria for the financial support. We also would like to thank the DIM analytics from the Region Ile de France for the founding.

\section{References}

[1] K. Demeestere, J. Dewulf, H. Van Langenhove, Heterogeneous photocatalysis as an advanced oxidation process for the abatement of chlorinated, monocyclic aromatic and sulfur volatile organic compounds in air: State of the art, Crit. Rev. Environ. Sci. Technol. 37 (2007) 489-538.

[2] M. A. Oturan, J.J. Aaron, Advanced oxidation processes in water/wastewater treatment: Principles and applications. A review, Crit. Rev. Environ. Sci. Technol. 44 (2014) 2577-2641.

[3] M. Cheng, G.M. Zeng, D.L. Huang, C. Lai, P. Xu, C. Zhang, Y. Liu, Hydroxyl radicals based advanced oxidation processes (AOPs) for remediation of soils contaminated with organic compounds: A review, Chem. Eng. J. 284 (2016) 582-598. 
[4] P. Bautista, A.F. Mohedano, J.A. Casas, J.A. Zazo, J.J. Rodriguez, An overview of the application of Fenton oxidation to industrial wastewaters treatment, J. Chem. Technol. Biotechnol. 83 (2008) 13231338.

[5] M. Brienza, M. Mahdi Ahmed, A. Escande, G. Plantard, L. Scrano, S. Chiron, S.A. Bufo, V. Goetz, Use of solar advanced oxidation processes for wastewater treatment: Follow-up on degradation products, acute toxicity, genotoxicity and estrogenicity, Chemosphere 148 (2016) 473-480.

[6] J.M. Poyatos, M.M. Muñio, M.C. Almecija, J.C. Torres, E. Hontoria, F. Osorio, Advanced Oxidation Processes for wastewater treatment : State of the art, Water Air Soil Pollut. 205 (2010) 187-204.

[7] A.D. Bokare, W. Choi, Review of iron-free Fenton systems for activating $\mathrm{H}_{2} \mathrm{O}_{2}$ in advanced oxidation processes, J. Hazard. Mater. 275 (2014) 121-135.

[8] M. Coste, C. Batbedat, C. Feliers, D. Olejnik, J. Cigana, P. Cervantes, Refractory COD removal in the chemical industry: technico-economic comparison of advanced oxidation processes, in Proceeding of WEFTEC'03, $76^{\text {th }}$ Annual Technical Exhibition and Conference, Los Angeles, United States,11-15 October 2003.

[9] E.S. Elmolla, M. Chaudhuri, Comparison of different advanced oxidation processes for treatment of antibiotic aqueous solution, Desalination 256 (2010) 43-47.

[10] A.C. Affam, M. Chaudhuri, S.R.M. Kutty. Comparison of Five Advanced Oxidation Processes for Degradation of Pesticide in Aqueous Solution, Bull. Chem. React. Eng. Catal. 13 (2018) 179-186.

[11] B.W. Tyre, R.J. Watt, G.C. Miller, Treatment of four biorefractory contaminants in soils using catalyzed hydrogen peroxide, J. Environ. Qual. 20 (1991) 832-838

[12] M. Hartmann, S. Kullmann, H. Keller, Wastewater treatment with heterogeneous Fenton-type catalysts based on porous materials, J. Mater. Chem. 20 (2010) 9002-9017.

[13] A. Ni Soon, B.H. Hameed, Heterogeneous catalytic treatment of synthetic dyes in aqueous media using Fenton and photo-assited Fenton process, Desalination 269 (2011) 1-16.

[14] A. Dhakshinamoorthy, S. Navalon, M. Alvaro, H. Garcia, Metal nanoparticles as heterogeneous Fenton catalysts, Chem. Sus. Chem. 5 (2012) 46-64. 
[15] N. Ferroudj, J. Nzimoto, A. Davidson, D. Talbot, E. Briot, V. Dupuis, A. Bée, M.S. Medjram, S. Abramson, Maghemite nanoparticles and maghemite/silica nnanocomposite microspheres as magnetic Fenton catalysts for the removal of water pollutants, Appl. Catal. B 136-137 (2013) 9-18.

[16] A. Bafana, S.S. Devi, T. Chakrabarti, Azo dyes: past, present and the future, Environ. Rev. 19 (2011) 350-370.

[17] C.M. Santana, Z.S. Ferrera, M.E. Padron, J.J. Rodriguez, Methodologies for the extraction of phenolic compounds from environmental samples: new approaches, Molecules 14 (2009) 298-320

[18] P. Kovacic, R. Somanathan, Toxicity of imine-iminium dyes and pigments: electron transfer, radicals, oxidative stress and other physiological effects, J. Appl. Toxicol. 34 (2014) 825-834.

[19] Y. Li, F.F. Zhang, X.M. Liang A. Yediler, Chemical and toxicological evaluation of an emerging pollutant (enrofloxacin) by catalytic wet air oxidation and ozonation in aqueous solution, Chemosphere 90 (2013) 284-291.

[20] A. Jing Li, O.J. Schmitz, S. Stephan, C. Lenzen, P. Yin-Kit Yue, K. Li, H. Li, K. Sze-Yin Leung, Photocatalytic transformation of acesulfame: Transformation products identification and embryotoxicity study, Water Res. 89 (2016) 68-75.

[21] M.S. Dieckmann, K.A. Gray, A comparison of the degradation of 4-nitrophenol via direct and sensitized photocatalysis in $\mathrm{TiO}_{2}$ slurries, Wat. Res. 30 (1996) 1169-1183.

[22] A. Houas, H. Lachheb, M. Ksibi, E. Elaloui, C. Guillard, J.-M. Herrmann, Photocatalytic degradation pathway of methylene blue in water, Appl. Catal. B 31 (2001) 145-157.

[23] T. Zhang, T. Oyama, A. Aoshima, H. Hidaka, J. Zhao, N. Serpone, Photooxidative N-demethylation of methylene blue in aqueous $\mathrm{TiO}_{2}$ dispersions under UV irradiation, J. Photochem. Photobiol. A 140 (2001) 163-172.

[24] C. Baiocchi, M.C. Brussino, E. Pramauro, A.B. Prevot, L. Palmisano, G. Marci, Characterization of methyl orange and its photocatalytic degradation products by HPLC/UV-VIS diode array and atmospheric pressure ionization quadrupole ion trap mass spectrometry, Int. J. Mass Spectrom. 214 (2002) 247-256. 
[25] A. Di Paola, V. Augugliaro, L. Palmisano, G. Pantaleo, E. Savinov, Heterogeneous photocatalytic degradation of nitrophenols, J. Photochem. Photobiol. A 155 (2003) 207-214.

[26] I.K. Konstantinou, T.A. Albanis, $\mathrm{TiO}_{2}$-assited photocatalytic degradation of azo dyes in aqueous solutions: kinetic and mechanistic investigations. A review, Appl. Catal. B 49 (2004) 1-14.

[27] H. Gnaser, M.R. Savina, W.F. Calaway, C.E. Tripa, I.V. Veryovkin, M.J. Pellin, Photocatalytic degradation of methylene blue on nanocristalline $\mathrm{TiO}_{2}$ : Surface mass spectrometry of reaction intermediates, Int. J. Mass Spectrom. 245 (2005) 61-67.

[28] K. Dai, H. Chen, T. Peng, D. Ke, H. Yi, Photocatalytic degradation of methyl orange in aqueous suspension of mesoporous titania nanoparticles, Chemosphere 69 (2007) 1361-1367.

[29] Y. He, F. Grieser, M. Ashokkumar, The mechanism of sonophotocatalytic degradation of methyl orange and its products in aqueous solutions, Ultrason. Sonochem. 18 (2011) 974-980.

[30] W. Li, D. Li, Y. Lin, P. Wang, W. Chen, X. Fu, Y. Shao, Evidence for the active species involved in the photodegradation process of methyl orange on $\mathrm{TiO}_{2}$, J. Phys. Chem. C 116 (2012) 3552-3560.

[31] C. Galindo, P. Jacques, A. Kalt, Photodegradation of the aminobenzene acid orange 52 by three advanced oxidation processes: $\mathrm{UV} / \mathrm{H}_{2} \mathrm{O}_{2}, \mathrm{UV} / \mathrm{TiO}_{2}$ and $\mathrm{VIS} / \mathrm{TiO}_{2}$. Comparative mechanistic and kinetic investigations, J. Photochem. Photobiol. A 130 (2000) 35-47.

[32] W. Zhang, X. Xiao, T. An, Z. Song, J. Fu, G. Sheng, M. Cui, Kinetics, degradation pathway and reaction mechanism of advanced oxidation of 4-nitrophenol in water by a $\mathrm{UV} / \mathrm{H}_{2} \mathrm{O}_{2}$ process, J. Chem. Technol. Biotechnol. 78 (2003) 788-794.

[33] B. Cercek, M. Ebert, Radiolytic Transients from p-nitrophenol and their inter- and intramolecular reactions, Adv. Chem. Ser. 81 (1968) 210-221.

[34] F. Follut, N. Karpel Vel Leitner, Radiolysis of aqueous 4-nitrophenol solution with $\mathrm{Al}_{2} \mathrm{O}_{3}$ or $\mathrm{TiO}_{2}$ nanoparticles, Chemosphere 66 (2007) 2114-2119.

[35] J. Gong, J. Wang W. Xie, W. Cai, Enhanced degradation of aqueous methyl orange by contact glow discharge electrolysis using $\mathrm{Fe}^{2+}$ as catalyst, J. Appl. Electrochem. 38 (2008) 1749-1755.

[36] L. Wei, H. Zhu, X. Mao, F. Gan, Electrochemical oxidation process combined with UV photolysis for the mineralization of nitrophenol in saline wastewater, Sep. Purif. Technol. 77 (2011) 18-25. 
[37] C. Ramirez, A. Saldaña, B. Hernandez, R. Acero, R. Gerra, S. Garcia-Segura, E. Brillas, J. PeraltaHernández, Electrochemical oxidation of methyl orange azo dye at pilot flow plant using BDD technology, J. Ind. Eng. Chem. 19 (2013) 571-579.

[38] T. Shirafuji, A. Nomura, Y. Hayashi, K. Tanaka, M. Goto, Matrix-assisted laser desorption ionization time-of-flight mass spectrometric analysis of degradation products after treatment of methylene blue solution with three-dimensionally integrated microsolution plasma. Jpn J. Appl. Phys. 55 (2016) 01AH02.

[39] Y. Du, M. Zhou, L. Lei, Role of the intermediates in the degradation of phenolic compounds by Fenton-like process, J. Hazard. Mater. B136 (2006) 859-865.

[40] C.S. Castro, M.C. Guerreiro, L.C.A. Oliveira, M. Gonçalves, A.S. Anastácio, M. Nazzarro, Iron oxide dispersed over activated carbon: Support influence on the oxidation of the model molecule methylene blue, Appl. Catal. A 367 (2009) 53-58.

[41] S.-P. Sun, A.T. Lemley, p-Nitrophenol degradation by a Heterogeneous Fenton-like reaction on nano-magnetite : Process optimization, kinetics, and degradation pathways, J. Mol. Catal. A 349 (2011) 71-79.

[42] C. Pulgarin, P. Peringer, P. Albers, J. Kiwi, Effect of Fe-ZSM-5 zeolite on the photochemical and biochemical degradation of 4-nitrophenol, J. Mol. Catal. A 95 (1995) 61-74.

[43] L. Gomathi Devi, S. Girish Kumar, K. Mohan Reddy, C. Munikrishnappa, Photo degradation of Methyl Orange an azo dye by Advanced Fenton Process using zero valent metallic iron: influence of various reaction parameters and its degradation mechanism, J. Hazard. Mater. 164 (2009) 459-467.

[44] A.A. Tireli, I.D. Guimares, J.C.D. Terra, R.R. da Silva, M.C. Geirreiro, Fenton-like processes and adsorption using iron oxide pillared clay with magnetic properties for organic compound mitigation, Environ. Sci. Pollut. R. 22 (2015) 870-881.

[45] M.A. Oturan, J. Peiroten, P. Chartrin, A.J. Acher, Complete destruction of p-nitrophenol in aqueous medium by Electro-Fenton method, Environ. Sci. Technol. 34 (2000) 3474-3479.

[46] E. Guivarch, S. Trevin, C. Lahitte, M.A. Oturan, Degradation of azo dyes in water by ElectroFenton process, Environ. Chem. Lett. 1 (2003) 38-44. 
[47] J.M. Joseph, H. Destaillats, H.-M. Hung, M.R. Hoffmann, The sonochemical degradation of azobenzene and related azo dyes: Rate enhancements via Fenton's reactions, J. Phys. Chem. A 104 (2000) 301-307.

[48] C. Sirtori, A. Agüera, I. Carra, J.A. Sanchez Peréz, Application of liquid chromatography quadrupole time-of-flight mass spectrometry to the identification of acetamiprid transformation products generated under oxidative processes in different water matrices, Anal. Bioanal. Chem. 406 (2014) 25492558.

[49] J.A. Sanchez Peréz, I. Carra, C. Sirtori, A. Agüera, B. Esteban, Fate of thiabendazole through the treatment of a simulated agro-food industrial effluent by combined MBR/Fenton processes at $\mu \mathrm{g} / \mathrm{L}$ scale, Water Res. 51 (2014) 55-63.

[50] R.R. Singh, Y. Lester, K.G. Linden, N.G. Love, G.E. Atilla-Gokcumen, D.S. Aga, Application of metabolite profiling tools and time-of-flight mass spectrometry in the identification of transformation products of iopromide and iopamidol during advanced oxidation, Environ. Sci. Technol. 49 (2015) 29832990.

[51] Y. Liu, X. He, X. Duan, Y. Fu, D. Fatta-Kassinos, D.D. Dionysiou, Significant role of UV and carbonate radical on the degradation of oxytetracycline in UV-AOPs: Kinetics and mechanism, Water Res. 95 (2016) 195-204.

[52] R. Massart, Preparation of Aqueous Magnetic Liquids in Alkaline and Acidic Media, IEEE Trans. Magn. MAG-17 (1981) 1247-1248.

[53] N. Andersson, R.W. Corkery, P.C.A. Alberius, One-Pot Synthesis of Well Ordered Mesoporous Magnetic Carriers, J. Mater. Chem. 17 (2007) 2700-2705.

[54] G. Zhan, H.C. Zeng, Charge-Switchable Integrated Nanocatalysts for Substrate-Selective Degradation in Advanced Oxidation Processes, Chem. Mater. 28 (2016) 4572-4582.

[55] J.T. Spadaro, L. Isabelle, V. Renganathan, Hydroxyl Radical Mediated Degradation of Azo Dyes: Evidence for Benzene Generation, Environ. Sci. Technol. 28 (1994) 1389-1393.

[56] M.K. Eberhardt, M. Yoshida, Radiation-Induced Homolytic Aromatic Substitution. I. Hydroxylation of Nitrobenzene, Chlorobenzene and Toluene, J. Phys. Chem. 77 (1973) 589-597. 
[57] L. Carlos, D. Fabbri, A.L. Capparelli, A.B. Prevot, E. Pramauro, F.S. García Einschlag, Intermediate Distributions and Primary Yields of Phenolic Products in Nitrobenzene Degradation by Fenton's Reagent, Chemosphere 72 (2008) 952-958. 
